\title{
1 Unbiased identification of trans regulators of ADAR and A-to-I RNA editing
}

3 Emily C. Freund ${ }^{1}$ Anne L. Sapiro ${ }^{1}$, Qin $\mathrm{Li}^{1}$, Sandra Linder ${ }^{1}$, James J. Moresco ${ }^{2}$, John R. Yates

$4 \quad \mathrm{III}^{2}$, Jin Billy $\mathrm{Li}^{1}$

$6{ }^{1}$ Department of Genetics, Stanford University, Stanford, CA, USA

$7 \quad{ }^{2}$ Department of Molecular Medicine, 10550 North Torrey Pines Road, SR302, The Scripps

8 Research Institute, La Jolla, California 92037

10 Correspondence: in.billy.li@stanford.edu

\section{Abstract}

14 Adenosine-to-Inosine RNA editing is catalyzed by ADAR enzymes that deaminate adenosine to 15 inosine. While many RNA editing sites are known, few trans regulators have been identified. We 16 perform BiolD followed by mass-spectrometry to identify trans regulators of ADAR1 and ADAR2

17 in HeLa and M17 neuroblastoma cells. We identify known and novel ADAR-interacting proteins.

18 Using ENCODE data we validate and characterize a subset of the novel interactors as global or 19 site-specific RNA editing regulators. Our set of novel trans regulators includes all four members 20 of the DZF-domain-containing family of proteins: ILF3, ILF2, STRBP, and ZFR. We show that 21 these proteins interact with each ADAR and modulate RNA editing levels. We find ILF3 is a

22 global negative regulator of editing. This work demonstrates the broad roles RNA binding

23 proteins play in regulating editing levels and establishes DZF-domain containing proteins as a 24 group of highly influential RNA editing regulators. 


\section{Introduction}

RNA editing is a widely conserved and pervasive method of mRNA modification in which the sequence of a mRNA is altered from that encoded by the DNA (Nishikura, 2016; Walkley and Li, 2017). In mammals, the most prevalent type of RNA editing is Adenosine-to-Inosine (A-to-I) RNA editing (Eisenberg and Levanon, 2018). After editing occurs, inosine is recognized by the cellular machinery as guanosine (G); therefore, the editing of a nucleotide can have a variety of effects, including altering RNA processing, changing splice sites, and expanding the coding capacity of the genome (Burns et al., 1997; Nishikura, 2010; Rueter et al., 1999). A-to-I editing is catalyzed by adenosine deaminase acting on RNA (ADAR) proteins, which are conserved in metazoans (Nishikura, 2016).

37 Humans have two catalytically active ADAR proteins, ADAR1 and ADAR2, that together are responsible for millions of RNA editing events across the transcriptome. ADAR1 primarily edits long, near-perfect double-stranded RNA regions that are formed by inverted repeats, predominantly Alu elements (Athanasiadis et al., 2004; Bazak et al., 2014; Blow et al., 2004; Levanon et al., 2004). These editing events have been shown to play a role in self versus nonself RNA recognition in the innate immune response, and thus dysregulation of ADAR1 leads to immune-related diseases such as Aicardi-Goutieres syndrome (AGS) (Blango and Bass, 2016;

44 Liddicoat et al., 2015; Mannion et al., 2014; Pestal et al., 2015; Rice et al., 2012). ADAR1 levels also correlate with tumor aggressiveness, as increases in ADAR1 editing suppress the innate immune response in tumors; accordingly, ADAR1 ablation helps with cancer therapy (Bhate et al., 2019; Gannon et al., 2018; Ishizuka et al., 2019; Liu et al., 2019; Nemlich et al., 2018). While the majority of ADAR1-regulated editing sites are found in repeat regions, ADAR2 is primarily responsible for editing adenosines found in non-repeat regions, particularly in the brain (Tan et al., 2017). ADAR2-regulated sites in non-repetitive regions include a number of editing events that alter the protein-coding sequences of neuronal RNAs, including GluR2, which encodes a

52 glutamate receptor in which RNA editing is necessary for its proper function. Further

53 demonstrating its important role in neuronal editing, dysregulation of human ADAR2 is

54 associated with multiple neurological diseases, including amyotrophic lateral sclerosis,

55 astrocytoma and transient forebrain ischemia (Slotkin and Nishikura, 2013; Tran et al., 2019).

56 Maintaining RNA editing levels is critical for human health, but how RNA editing levels are

57 regulated at specific editing sites across tissues and development is poorly understood. 
While RNA sequence and structure are critical determinants of editing levels, studies querying tissue-specific and developmental-stage-specific editing levels show that the level of editing at the same editing site can vary greatly between individuals and tissues. These changes do not always correlate with ADAR mRNA or protein expression, suggesting a complex regulation of editing events by factors other than ADAR proteins (Sapiro et al., 2019; Tan et al., 2017; Wahlstedt et al., 2009). Recently, an analysis of proteins with an RNA-binding domain profiled by ENCODE suggested that RNA-binding proteins play a role in RNA editing regulation. Further, in mammals, a small number of trans regulators of editing have been identified through functional experiments (Quinones-Valdez et al., 2019). Some of these trans regulators of editing are site-specific, in that they affect editing levels at only a small subset of editing sites. These include RNA binding proteins such as DHX15, HNRNPA2/B1, RPS14, TDP-43, Drosha and Ro60 (Garncarz et al., 2013; Quinones-Valdez et al., 2019; Tariq et al., 2013). The recently identified ADAR binding partners, ELAVL1, DHX9 and SRSF9 have also been shown to affect the editing level of specific sites (Aktaş et al., 2017; Huang et al., 2018; Shanmugam et al., 2018; Stellos et al., 2016). In addition to site-specific regulators of editing, Pin1, WWP2 and AIMP2 have been shown to regulate editing through post-translational modification of the ADAR proteins (Behm et al., 2017; Marcucci et al., 2011; Tan et al., 2017). However, the complexity of editing level regulation across millions of editing sites in numerous tissues and developmental stages suggests that there are likely many other proteins that regulate editing.

Here, we take a unique approach to identify novel regulators of the ADAR proteins. We employ the BiolD system, which facilitates the biotinylation and subsequent purification of proteins that both transiently and stably interact with bait proteins (Roux et al., 2012), to uncover proteins that interact with ADAR1 and ADAR2 in two human cell lines, HeLa and BE(2)-M17 cells. Together, these experiments facilitate the identification of 269 ADAR-interacting proteins, 15 of which had been previously reported, and many of which we further validate using publicly available data. Interestingly, the top candidates for novel regulators of ADARs represent a family of proteins that all contain a DZF-domain: ILF3, ILF2, STRBP, and ZFR. These proteins interact with both

87 ADAR1 and ADAR2 in an RNA-dependent manner. We further characterize ILF3, the top

88 candidate, and find that it acts as a negative regulator of editing that binds RNA near editing

89 sites and globally regulates RNA editing levels. Furthermore, we demonstrate that the RNA

90 binding domains of ILF3 are necessary for its editing regulation. This class of DZF-domain-

91 containing proteins represents a novel group of RNA editing regulators and demonstrates the 
92 utility of the BiolD experiment as a tool to systematically identify ADAR-interacting proteins that

93 regulate RNA editing levels.

\section{Results}

\section{ADAR1 and ADAR2 BiolD identifies known and novel regulators of RNA editing}

RNA editing levels are dynamically regulated, but few proteins responsible for this regulation are known. We set out to identify novel regulators of the ADAR proteins by identifying the proteins that ADAR1 and ADAR2 interact within the cell. We hypothesized that proteins that transiently interact with ADAR might compete with or recruit ADAR at only subsets of mRNA loci, making them good candidates for site-specific regulators of RNA editing. To identify these proteins, we utilized the BiolD system (Roux et al., 2012). This method expands upon traditional immunoprecipitation (IP) and mass-spectrometry-based screening used to identify proteinprotein interactions. Traditional IPs enrich for proteins that form a stable complex with the bait protein but are less effective at identifying transient interactions. The BioID protocol efficiently captures both this transiently interacting class of regulators and the more standard stable protein partners by covalently labeling factors as they come into close proximity $(\sim 10 \mathrm{~nm})$ with a bait protein (Kim et al., 2014). These labeled proteins can then be immunoprecipitated and identified by mass-spectrometry (Figure 1A). To utilize this system, we fused ADAR proteins with a mutated form of the BirA biotin ligase (R118G), denoted BirA* (Kwon et al., 2000; Roux et al., 2012), which promiscuously and irreversibly biotinylates proteins in a proximity-dependent manner.

We lentivirally integrated either BirA*-ADAR1 or BirA*-ADAR2 constructs into two human cell lines, neuroblastoma BE(2)-M17 (M17) cells and HeLa cells. By using two cell lines, we hoped to identify both tissue-specific and universal editing regulators. To first demonstrate that the constructs were functional, we assessed editing levels at two editing sites. We observed an increase in editing at an ADAR1-specific site in PAICS upon expression of BirA*-ADAR1 and an

121 increase in editing at the ADAR2-specific site in GRIA2 upon expression of BirA*-ADAR2

122 compared to a BirA*-GFP control (Figure S1A), demonstrating that the BirA*-ADAR constructs 123 produce functional ADAR proteins. 
After verifying the activity of the BirA*-ADAR proteins, we performed the BiolD experiments. We produced three biological replicates for both BirA*-ADAR1 and BirA*-ADAR2 in both M17 and HeLa cells, along with three replicates of two different negative controls: nuclear localized BirA*GFP and cells with no transgene and thus no BirA* expression in each cell line (Figure 1B). The cells were incubated overnight with D-biotin to allow the fusion proteins to biotinylate interactors. We then performed a stringent denaturing pulldown with streptavidin agarose and analyzed the elution by mass spectrometry (see Methods for details). To identify the putative ADAR1- and ADAR2-interacting proteins from our list of all proteins returned from the massspectrometry, we determined the fold change in peptide counts for each protein in each BirA*ADAR condition compared to BirA*-GFP and the no BirA* controls, then defined hits as those proteins having at least two-fold enrichment over both of the negative control conditions in at least two biological replicates (Figure 1C, S3A). In total, we identified 269 proteins as putative interactors of either ADAR1 and/or ADAR2 in HeLa and/or M17 cells. When comparing across all 4 conditions, we found hits unique to each condition: 26 proteins unique to ADAR 1 and 201 unique to ADAR2, 127 proteins unique to Helas and 118 unique to M17 cells, and 57 proteins shared across multiple conditions (Figure 1D, S3B,C). Supporting the efficacy of this screening approach, we found many of the proteins previously reported to affect editing or interact with ADAR proteins. The ADAR-interacting proteins we identified included DHX15, RPS14, and ELAVL1, which were previously reported to regulate editing at specific sites, and SFPQ, HNRNPH1 and PTBP1, which are known post-transcriptional regulators of ADARs (Hirose et al., 2014; Yang et al., 2015). We also identified known ADAR-interacting protein CPSF6 and proteins involved in $\mathrm{L} 1$ Line element retrotransposition that were previously found in a complex with ADAR1: DHX15, SFPQ, NCL, TUBB, NONO, HSPA8, SF3B1, and HNRNPL (Binothman et al., 2017; Orecchini et al., 2017). Furthermore, we identified proteins that, like ADAR, bind the ACA11 transcript: SF3B1, PARP1, NCL, DDX21 and ILF3 (Figure 1D, S3C) (Chu et al., 2012).

Overall, we found many more candidates specific to ADAR2 than ADAR1, which is likely due to the fact that ADAR2-BirA* was more highly expressed than ADAR1-BirA*; we found that overexpressed ADAR2 consistently accumulates to higher protein levels than overexpressed ADAR1, perhaps reflecting different mechanisms of regulation (data not shown). We also found a large number of cell-type-specific ADAR interactors (118 M17 versus $127 \mathrm{HeLa}$ hits), which we hypothesized might be differentially expressed between the two cell types. To determine whether cell-type-specific interactors were more highly expressed in the cell type in which they were identified, we measured the expression level of our candidates in each cell line using 
RNA-seq. We found that candidates specific to M17 cells were more highly expressed in M17 cells than in HeLa cells (as measured by FPKM, Wilcoxon test, $p<0.001$ ), but the reverse was not true, suggesting that differential mRNA expression does not fully explain the specificity of proteins interacting with ADARs in these two cell types (Figure S3A,B).

\section{Novel ADAR-interacting proteins bind near editing sites to alter editing levels}

Many proteins found in the BiolD screen were RNA binding proteins and RNA processing enzymes, suggesting enrichment for biologically relevant proteins. We utilized publicly available RNA-seq data from shRNA knockdowns performed in K562 cells by the ENCODE project (Sundararaman et al., 2016) to identify whether any of the RNA binding proteins identified in the BiolD altered editing levels. We found that knockdown of 8 of the 19 proteins profiled by ENCODE resulted in increases or decreases in the editing levels of more than 100 sites, while the remaining 11 affected a smaller more specific set of sites (Figure 2A, 2S). We hypothesized that RNA-binding proteins that affected editing might bind RNA near editing sites and alter ADAR binding and therefore editing levels at those sites. To test whether this was true of our 19 candidate interactors, we utilized the publicly available eCLIP-seq data ("A Large-Scale Binding and Functional Map of Human RNA Binding Proteins | bioRxiv," n.d.) to identify the RNA binding sites of the 19 candidates profiled by ENCODE. We determined the proximity of each protein's RNA binding sites to known editing sites. Of these 19 candidates, 4 showed evidence of binding at editing sites (Figure 2B) and 9 showed evidence of binding nearby, but not right at, editing sites (Figure 2C). This is consistent with the hypothesis that these RBPs might interfere with or recruit ADARs to these editing sites. The remaining 6 candidates did not show evidence of binding near editing sites (Figure 2D). Included in this last group is PTBP1, which has been shown to be a translational regulator of ADAR (Yang et al., 2015); this role may explain why it physically interacts with ADAR but does not bind near editing sites. For the 13 RBPs that bound RNA at or near editing sites, we compared the positions of their binding sites to the editing sites that they regulated to determine whether their knockdown affected editing levels specifically at the sites that were bound by the proteins. We found a statistical enrichment for 11 of the 13 candidates indicating that they changed editing levels at editing sites near where they bound RNA, suggesting a direct role in RNA editing (Figure 2E). Of those proteins, only U2AF2 and XRCC6 altered the majority of known editing sites in a single direction (Figure 2A,E), suggesting that RBPs binding at or near editing sites does not necessarily lead to the same changes in editing levels at every site. 


\section{DZF-domain-containing proteins interact with ADAR1 and ADAR2 in an RNA-dependent} manner

One group of proteins that we identified in the BiolD were four related proteins, ILF2, ILF3, STRBP and ZFR, which are the only four human proteins that contain a DZF domain. The DZF domain (domain associated with zinc fingers) is a poorly understood domain, but it has been

200 shown to drive protein dimerization and facilitate RNA binding (Castello et al., 2016; Wolkowicz 201 and Cook, 2012). Two of these DZF-domain-containing proteins, ILF3 and ZFR, were the only proteins identified as hits in all four BiolD conditions (ADAR1- and ADAR2-interactors in both cell types). ILF3 has been shown previously to interact with ADAR1-p150 in an RNA-dependent manner in the cytoplasm (Nie et al., 2005). Our results extend its interaction to ADAR-p110, the isoform we overexpressed in our BiolD experiments. Another DZF-domain-containing protein, STRBP, was identified in three out of four conditions (all except HeLa BirA*ADAR1) and the fourth, ILF2, was identified in the M17 BirA*-ADAR1 condition, strongly implicating DZF-domaincontaining proteins as ADAR-interacting proteins. In addition to a DZF domain, all three proteins except for ILF2 contain double-stranded RNA binding domains (dsRBDs), similar to ADAR1 and ADAR2 (Figure 3A). ILF3 and STRBP have two dsRBDs, while ZFR has three widely spaced

211 zinc finger domains, which are thought to mediate interaction with dsRNAs. Intriguingly, ILF3's

212 dsRBDs have been shown to be structurally similar to those of ADAR2 and to compete for

213 similar binding sites in vitro (Wolkowicz et al 2012). In addition, ILF3 has been shown to

214 regulate circular RNA biogenesis by binding near the highly edited Alu elements (Li et al., 2017).

215 Because this entire class of proteins was highly enriched in the BiolD hit list and had been

216 previously suggested to interact with ADAR1, we chose to further characterize this class of

217 proteins to understand their regulation of RNA editing.

To validate the interaction between ILF3, ZFR, STRBP, and ILF2 and each ADAR protein, we 220 performed traditional co-IPs in M17 cells. We transduced a FLAG-tagged ADAR1, ADAR2, or

221 GFP as a negative control, into M17 cells (Figure S5A, B) and performed an IP using an

222 antibody against FLAG. To further determine whether the interaction between each ADAR and 223 each DZF-domain-containing candidate was dependent on RNA, we treated half of each of the

224 IPs with RNase A. Whereas FLAG-GFP did not immunoprecipitate any of the four candidates,

225 FLAG-ADAR1 and FLAG-ADAR2 immunoprecipitated each candidate. In all cases, the 226 interaction between each candidate and each ADAR was decreased upon addition of RNase A, 
227 suggesting that their interaction is at least partially RNA-dependent, and that they do not form a

228 stable complex off of RNA (Figure 3B, C).

229

230 We also wanted to interrogate the reciprocal condition, the ability of each candidate to

231 immunoprecipitate each ADAR protein; however, M17 and HeLa cells do not express high

232 levels of ADAR2, making it difficult to analyze endogenous ADAR2's interaction with each

233 candidate in these cells. To address this problem, we created an M17 cell line overexpressing

234 ADAR2 by lentiviral transduction. We then stably expressed a FLAG-tagged version of each

235 candidate, or FLAG-GFP as a negative control, in the ADAR2-overexpressing M17 cell line

236 (M17-ADAR2-OE) (Figure S5C-F). We performed FLAG IPs with and without RNase A

237 treatment in this cell line. We used pulldown of ILF2 as a positive control because previous work

238 established that it binds each of the other DZF-domain-containing proteins in an RNA-

239 independent manner, and thus we would expect to see that it binds each candidate protein with

240 and without RNase A (Wolkowicz and Cook, 2012). As expected, ILF2, ILF3, and ZFR all bound

241 ADAR1 and ADAR2 in an RNA-dependent manner and bound ILF2 independent of RNA

242 (Figure 3D-G). Unexpectedly, in contrast to the FLAG-ADAR2 IP, in the FLAG-STRBP IP we

243 found an RNA-independent interaction between STRBP with ADAR2 (Figure 3G), which could

244 indicate a different mode of interaction depending on the bait protein. Taken together, we were

245 able to fully validate that three of our top candidates biochemically interact with ADARs in an

246 RNA-dependent manner.

\section{DZF-domain-containing proteins affect A-to-I RNA editing}

Similar to the validation of other candidates that we performed with publicly available RNA-seq and eCLIP-seq data, we wanted to perform a more thorough analysis of the role of DZFdomain-containing proteins in A-to-I RNA editing. We transiently overexpressed ILF2, ILF3, and STRBP in HEK293T cells and in HEK293T cells stably overexpressing ADAR2 through lentiviral

254 transduction (HEK293T-ADAR2-OE) (Figure 4A, B); as in M17 cells, ADAR2 is lowly expressed

255 in HEK293T cells, thus requiring overexpression for analysis of ADAR2-controlled editing sites.

256 We choose to perform these experiments in HEK293T cells both because in contrast to M17

257 cells, they were suitable for transient transfection and it extended our findings to a third cell

258 type. We first verified that each protein was overexpressed at the transcript level (Figure 4A, B)

259 and protein level (Figure S6A,B). To examine the effects of the DZF-domain-containing

260 proteins on editing levels, we used microfluidic multiplex PCR and sequencing (mmPCR-seq), in 
261 which we PCR-amplified cDNA at thousands of known editing sites for subsequent Illumina

262 sequencing [REF]. mmPCR-seq editing level measurements were highly reproducible between

263 two biological replicates from each cell type (Figure S7A, Table S1). We compared the editing

264 levels at these highly reproducible sites between the GFP-overexpressing control and the cells

265 overexpressing DZF-domain-containing proteins in both HEK293T and HEK293T-ADAR2-OE

266 backgrounds. Overexpression of ILF2 had a moderate effect in both HEK293T and HEK293T-

267 ADAR2-OE cells (Figure 4C, D); 11 sites had reduced editing in HEK293T cells, while 15 sites

268 showed increased editing, and 18 sites showed reduced editing in HEK293T-ADAR2-OE cells.

269 This relatively weak and bidirectional effect on editing is consistent with ILF2's identification in

270 only one of the four BiolD conditions (interacting with ADAR1 in M17 cells), suggesting that on

271 its own it may not be a robust ADAR interactor. STRBP demonstrated a slightly stronger effect;

27219 sites were reduced in HEK293T cells and 27 in HEK293T-ADAR2-OE cells with only 7 and 3

273 sites increased in editing respectively (Figure 4E, F). By far, ILF3 demonstrated the strongest

274 effect; 39 sites were decreased in HEK293T cells and 47 in HEK293T-ADAR2-OE cells with

275 only 4 and 1 editing sites showing increased editing levels, respectively (Figure 4G, H). To

276 better understand the role of ILF3 in editing regulation, we performed RNA-seq on cells with

277 ILF3 overexpression in both HEK293T and HEK293T-ADAR2-OE backgrounds. Similar to

278 mmPCR-seq, editing level measurements were highly reproducible between two biological

279 replicates from each cell type (Figures S7B, Table S2). This more expansive genome-wide

280 analysis further revealed ILF3 to be a global regulator of editing, as almost the entire editing

281 landscape was reduced when ILF3 was overexpressed. We observed 221 sites with a

282 significantly reduced editing level ( $p<0.05$, Fisher's exact test) compared to only 16 with an

283 increased editing level in HEK293T cells, and we found 237 sites with a significantly reduced

284 editing level compared to only 2 with an increased editing level in HEK293T-ADAR2-OE cells

285 (Figure 4I). In addition, we calculated overall editing as the total number of edited G reads over

286 the total of $A+G$ reads at all known editing sites combined as a global measure of ADAR

287 activity (Tan et al 2017). We found that in both HEK293T and HEK293T-ADAR2-OE cells,

288 overall editing was significantly reduced, from $2.43 \%$ to $1.45 \%$ in HEK293T cells, and from

$2894.56 \%$ to $2.45 \%$ in HEK293T-ADAR2-OE cells (Figure 4J). Overall, our data show that ILF3

290 and STRBP are both negative regulators of editing but that ILF3 is a stronger global regulator of

291 editing.

292

293 One possible explanation for the effect of DZF-domain-containing proteins on RNA editing could

294 be transcriptional or translational regulation of ADAR mRNA or protein levels. To test this 
hypothesis, we analyzed the mRNA and protein levels of ADAR1 and ADAR2 in ILF2-, ILF3-, and STRBP-overexpressing cells compared to GFP-overexpressing cells in HEK293T and HEK293T-ADAR2-OE cells. We found that ADAR1 mRNA and protein levels were largely unchanged (Figure S7A, B). ADAR2 transcript levels were reduced, which may account for some of the editing effects in HEK293T-ADAR2-OE cells (Figure S8A, B); however, there was a reduction in editing even in HEK293T cells, which largely lack ADAR2 expression and ILF2 overexpression showed a similar reduction in ADAR2 protein without a strong effect on editing, suggesting regulation of ADAR2 levels cannot be the sole mechanism that DZF-domaincontaining proteins use to repress editing.

RNA binding activity of ILF3 is necessary for its regulation of RNA editing

Because changes in ADAR levels did not explain the differences in editing that we observed, we hypothesized that the global regulator ILF3 and ADAR proteins compete for the same transcripts. This hypothesis is supported by previous work showing that ILF3 has structurally similar dsRBDs to ADAR2 (Wolkowicz and Cook, 2012). This mechanism would be consistent with our previously observed RNA-dependent interaction between ILF3 and the ADARs. In

312 addition, we and others have shown that ILF3 binds near editing sites in K562 cells (Figure 2B)

313 (Quinones-Valdez et al., 2019). If ILF3 competes for RNA binding sites with ADAR proteins, 314 then the ability of ILF3 to affect editing levels would be dependent on its ability to bind RNA. To 315 test this hypothesis, we overexpressed a FLAG-tagged mutant of ILF3 that lacks its two dsRBDs (4402-572) in HEK293T and HEK293T-ADAR2-OE cells (Figure 5A, B, S9A, Table

317 S1). In both cases, ILF3- $\triangle$ dsRBD-OE did not induce a global reduction in editing (Figure 5C, 318 S9B), suggesting that ILF3's RNA binding ability is necessary for its regulation of editing levels. 319 In addition, we performed a FLAG IP to pull down the mutant and wild-type version of ILF3 and 320 found that only wild-type ILF3 interacts with ADAR1 and ADAR2 (Figure 5D). This result further 321 supports the hypothesis that it is the ability of ILF3 to bind RNA and compete for substrates with 322 ADARs that regulates editing levels.

323

324 Discussion

325

326 RNA editing is widely conserved and pervasive, leading to changes at the RNA level. It is 327 catalyzed by two enzymatically active ADARs, ADAR1 and ADAR2. Expression levels of these 328 ADAR enzymes do not correlate with the editing frequency of large classes of sites (Tan et al., 
2017), which strongly suggests the presence of additional editing regulators. We performed a large-scale, unbiased assay to systematically identify novel editing regulators in humans by employing a biochemical-based screening approach, BiolD. BiolD labels proteins in proximity of

332 a BirA*-tagged bait protein, thus allowing us to identifying proteins that physically interact with

333 ADARs transiently or stably. As editing regulation is known to be cell-type-specific, we

334 performed the BiolD screen in two different cell types, HeLa and M17, using either ADAR1 or ADAR2 as bait. This approach was highly successful in enriching for ADAR interactors, in that it identified most previously known ADAR binding partners and editing regulators. The small number of previously known ADAR-interactors that were not found here probably interact with ADAR proteins in a cell-type-specific context that may not exist in our system. While many proteins were found in multiple BioID conditions, supporting the reproducibility and robustness of this approach, we also uncovered numerous cell-type-specific and ADAR1- or ADAR2specific interactors. Cell-type-specific hits may arise from differences in expression of those proteins between cell types, however a similar explanation would not explain ADAR-specific hits. We cannot rule out that some of the proteins specific to each condition may be an artifact of the common variability seen in mass-spectrometry-based screens; however, the reproducibility of the data suggests strong biological signals in the dataset. This dataset is highly complementary to and greatly extends a recently published study of RNA editing regulators identified through analysis of the ENCODE RNA binding protein knockdown RNA-seq dataset (Quinones-Valdez et al., 2019). We have uncovered 269 proteins that interact with ADARs, many of which overlap with the recently identified RNA binding proteins that regulate editing, validating our approach. Our biochemical screen enabled us to identify additional regulators, many of which are potentially ADAR and cell-type-specific.

When we first set out to identify trans regulators of editing, we were investigating two major potential mechanisms that could account for the observed tissue-specific differences in editing. Trans regulators could act directly on ADAR proteins to modify their activity at all sites equally 356 but be differentially expressed in different tissues. Alternatively, trans regulators could affect a 357 subset of sites by only interacting with or regulating ADAR proteins at those sites. We used 358 publicly available RNA-seq and eCLIP-seq data from the ENCODE project to determine that we 359 had uncovered both global and site-specific RNA editing regulators. Specifically, we found that 360 the majority of our hits profiled by the ENCODE project showed binding at or near editing sites, 361 and knocking down those proteins resulted in changes in the editing levels specifically at the 362 sites they bound. This finding suggests that many of the editing sites regulated in trans are 
controlled by proteins interacting with ADAR proteins at editing sites. In some cases, the primary function may not be to regulate editing, but they nevertheless alter ADAR binding and editing. For example, BiolD recovered many of the proteins found in paraspeckles, a complex ADAR1 had previously been found to be associated with (Anantharaman et al., 2016),

367 highlighting the power of BiolD to identify nearly all proteins found in a complex. We also found 368 proteins that are ADAR interactors but not regulators of editing. These proteins may help ADAR 369 proteins perform their other known functions in the cell such as miRNA regulation, or as yet 370 unknown editing-independent functions of ADARs. They may also regulate the translation of 371 ADARs, similar to PTBP1, which can activate the translation of ADAR-p110 through an IRES-

372 like element (Yang et al., 2015).

373

The most intriguing finding of the BiolD screens was the identification of all four DZF-domain containing proteins among the strongest hits. This finding suggests the importance of this class of proteins as ADAR interactors. These proteins largely interact with ADARs in an RNAdependent fashion. ILF3 and STRBP appear to compete with ADAR to bind and edit dsRNAs because overexpression of these proteins led to decreased editing overall. In particular, ILF3 inhibited editing at a large number of sites, suggesting that it is a strong global suppressor of editing. This finding is consistent with a recent report that knockdown of ILF3 decreases editing at specific sites in K562 cells (Quinones-Valdez et al., 2019). Because ILF3 and ADAR2 have structurally similar dsRBDs and are able to compete for substrates in a biochemical assay (Wolkowicz and Cook, 2012) we tested whether this mechanism held in cells. We overexpressed a mutant version of ILF3 that lacks both dsRNA binding domains and found that the mutant did not interact with either ADAR and was unable to suppress editing. As we found that a number of RNA binding proteins bound RNA near the editing sites that they regulated, we further hypothesize that competing with ADAR for RNA binding is a prominent regulatory mechanism of RNA binding proteins on editing levels. It will be interesting to more thoroughly explore the role of the other DZF domain containing 391 proteins, ZFR and STRBP, in interacting with ADARs and regulating editing. The companion 392 paper from our lab shows that Zinc finger RNA binding protein Zn72D, the fly homolog of ZFR, 393 regulates over half of editing events in the fly brain, and that knockdown of ZFR leads to a 394 decrease in a large number of editing events in mouse primary neurons. Zn72D also interacts 395 with dADAR in an RNA-dependent manner, suggesting a similar mechanism to ILF3 regulation 396 of ADARs that we detail here, but ZFR appears to be a positive regulator of editing, in contrast 
397 to ILF3. ZFR regulates editing of primarily ADAR2 editing sites in mouse primary neurons,

398 suggesting that ZFR may be a particularly important regulator of ADAR2 editing in the brain

399 (Sapiro et al., n.d.). Future work may explore whether STRBP has a similarly strong tissue-

400 specific effect on editing, as it is highly expressed in the brain and testes. Together, our work

401 suggests that DZF-domain-containing proteins as a class are critical for proper RNA editing, and

402 future work is needed to further explore the mechanistic details and cell-type specificity of this

403 role, including the potential role of the actual DZF-domain in supporting a protein interaction with

404 ADAR.

405

406 The BiolD experiments uncovered a large number of novel ADAR interactors and putative RNA

407 editing regulators. These hits can be further characterized for their roles in both RNA editing

408 and/or non-canonical roles for ADAR in the cell. Such knowledge may enable the manipulation

409 of editing levels at specific sites without the manipulation of ADAR proteins themselves, which

410 may have therapeutic benefit for cancer, autoimmune diseases, neurological diseases, or other

411 diseases in which ADARs play a role. 


\section{Methods}

\section{Cell Culture, Transfections and Viral Transductions}

416 HeLA S3 and 293T cells were cultured in DMEM supplemented with 10\% FBS and Pen/Strep.

417 M17 cells were cultured in F12 media supplemented with 10\% FBS and Penn/Strep. Lentivirus

418 was generated using standard methods: briefly, 293T cells were transfected with $3^{\text {rd }}$ generation

419 packaging constructs and target plasmid for 8 hours then media was changed and viral

420 supernatant was collected at 24 and 48 hour after and filtered at .45 uM. Cells were transduced

421 with $1 X$ viral supernatants supplemented with $5 \mathrm{ug} / \mathrm{ml}$ polybrene. Media was changed 12-24

422 hours later and selection began with the appropriate antibiotics between days 3-7.

424 BirA* constructs (BirA*-ADAR1, BirA*-ADAR2, BirA*-GFP) were created by subcloning the 425 cDNA for each gene into the pCDH backbone containing a 3XFLAG tag, a nuclear localization 426 sequence and the BirA* cDNA sequence upstream of the target gene, resulting in 3xFLAG-NLS427 BirA*-ADAR1, 3xFLAG-NLS-BirA*-ADAR2, and 3xFLAG-NLS-BirA*-GFP constructs and, when 428 translated, fusion proteins. These constructs were used to generate lentivirus, as described 429 above.

431 FLAG-tagged constructs for ADARs (ADAR1 and ADAR2) and DZF-domain-containing proteins 432 (ILF2, ILF3, STRBP, ZFR) were generated by subcloning the cDNA for each gene into the 433 pCDH backbone containing an upstream ( $\mathrm{N}$-terminal) 3xFLAG. These constructs were used to 434 generate lentivirus, as described above.

A non-tagged version of ADAR2 (for Figure 3) was generated by subcloning the ADAR2 cDNA 437 into the pCDH backbone.

All transient transfection constructs were generated by subcloning the cDNA of interest into the pCDNA 3.1-3xFLAG (GFP, ADAR1, ADAR2, ILF2, ILF3, STRBP). All transient transfections were performed with Lipofectamine 2000 according to manufacturer's protocol.

443 ILF3 $\triangle$ dsRBD was generated by synthesizing an ILF3 cDNA lacking the nucleotides coding for 444 amino acids 402-572 (inclusive) and subcloning it into pCDNA 3.1-3xFLAG and pCDH. 
447 Each of the three (ADAR1, ADAR2, and GFP) BirA constructs were stably expressed in HeLa

448 and M17 cells via lentiviral transduction. Ten 150mm plates of confluent cells expressing each

449 construct were grown overnight in media with a final concentration of $50 \mathrm{uM}$ D-Biotin (Life

450 technologies, B-20656).

451

\section{$452 \quad$ Nuclear Lysate Preparation}

453 Cells were harvested, pelleted and washed once with 1 X PBS. A 10X pellet volume of ice cold 454 cytoplasmic extraction buffer (10 mM Hepes pH 7.5, $10 \mathrm{mM} \mathrm{KCL}, 1.5 \mathrm{mM} \mathrm{MgCl2,} 0.34 \mathrm{M}$

455 Sucrose, 10\% glycerol) with $1 \mathrm{mM}$ DTT and protease inhibitors (cOmplete, Mini, EDTA-free

456 Protease Inhibitor Cocktail Tablets, \# 4693159001) was added and the pellet was gently

457 resuspended. Cells were incubated on ice for $15 \mathrm{~min}$. Triton X-100 was added to a final

458 concentration of $0.1 \%$ and cells were vortexed for 10 seconds then incubated on ice for $5 \mathrm{~min}$.

459 Cells were spun down at $1300 x g$ for $5 \mathrm{~min}$ at $4 \mathrm{C}$, and washed once with cytoplasmic extraction

460 buffer, spun down again and the supernatant was discarded. The nuclei were lysed with $7 X$ the 461 volume of the original pellet with high salt NP-40 lysis buffer (25 mM Hepes pH 7.5, $420 \mathrm{mM}$

$462 \mathrm{NaCl}, 1.5 \mathrm{mM} \mathrm{MgCl} 2,10 \%$ glycerol, 0.5\% NP-40) and incubated on ice for 20 min with

463 occasional vortexing. Lysate was then spun at $>20,000 \times \mathrm{xg}$ for $15 \mathrm{~min}$ at $4 \mathrm{C}$. Protein

464 concentration was then measured by BCA (Pierce, 23227).

\section{Preclear Lysate}

$4671 \mathrm{~mL}$ of Agarose Control Resin (Pierce, 26150) slurry was added per sample to a $10 \mathrm{~mL}$ 468 centrifuge column (Pierce, 89898) and placed inside a $50 \mathrm{~mL}$ conical tube and spun at $1000 \mathrm{xg}$ 469 for $1 \mathrm{~min}$ to remove storage buffer. The resin was washed once with $4 \mathrm{X}$ resin bed volume of 470 high salt NP-40 lysis buffer, and spun at 1000xg for $1 \mathrm{~min}$. Nuclear lysate was added to the 471 washed resin and incubated at $4 \mathrm{C}$ for $>2 \mathrm{hrs}$, with rotation. The resin was placed in a new $50 \mathrm{~mL}$ 472 conical, and spin at 1000xg for 1 min to collect the precleared lysate. The protein concentration

473 is measured by BCA (Pierce, 23227).

474

475 Bind Biotinylated targets to Streptavidin beads

$47620 \mathrm{uL}$ of High Capacity Streptavidin Agarose (Pierce, 20359) per $1 \mathrm{mg}$ of lysate is added to a 10

$477 \mathrm{ml}$ centrifuge column placed inside a $50 \mathrm{~mL}$ conical tube. 6 volumes of high salt NP-40 lysis

478 buffer is added to column and allowed to drain by gravity flow. Nuclear lysate is added to

479 washed resin and incubated at $4{ }^{\circ} \mathrm{C}$ overnight, with rotation. 
482 Eluate was collected by gravity flow and retained as the depleted fraction. The resin was then

483 stringently washed twice with 10X volumes of the resin bed volume of high salt NP-40 lysis

484 buffer with $0.3 \%$ SDS and then twice with high salt NP-40 lysis buffer with $1.0 \%$ SDS. The

485 washed resin was resuspended in $900 \mathrm{ul}$ of elution buffer (1XPBS, 5\% SDS, $10 \mathrm{mM}$ D-Biotin

486 (Life technologies, B-20656), transferred to an eppendorf tube and boiled for $15 \mathrm{~min}$. The resin

487 is then placed in a $2 \mathrm{~mL}$ centrifuge column (Pierce, 89896) placed inside a $15 \mathrm{~mL}$ conical tube

488 and spun at $1000 \mathrm{xg}$ for $1 \mathrm{~min}$ to collect the eluate. $\mathrm{MeOH} / \mathrm{Chloroform}$ precipitation was used to

489 concentrate the eluate. In brief, to $150 \mu \mathrm{L}$ of eluate $600 \mu \mathrm{L}$ of methanol was added followed by

$490150 \mu \mathrm{L}$ of chloroform and vortexed. $450 \mu \mathrm{L}$ of ultrapure water was added and vortexed

491 then centrifuged at $14,000 \mathrm{xg}$ for $5 \mathrm{~min}$. Upper aqueous phase was removed and $450 \mu \mathrm{L}$ of

492 methanol, $1 \mathrm{uL}$ GlycoBlue was added and vortexed. The protein was pelleted by centrifuging at

$49314,000 x g$ for $5 \mathrm{~min}$ and then methanol removed completely. Pellets were resuspended in $25 \mathrm{ul}$

494 of $1 \times$ SDS-PAGE sample buffer then boiled at $95 \mathrm{C}$ for $5 \mathrm{~min}$.

\section{BiolD Mass Spectrometry and Analysis}

497 Reagents and Chemicals

498 Deionized water was used for all preparations. Buffer A consists of $5 \%$ acetonitrile $0.1 \%$ formic 499 acid, buffer $B$ consists of $80 \%$ acetonitrile $0.1 \%$ formic acid, and buffer C consists of $500 \mathrm{mM}$ 500 ammonium acetate $0.1 \%$ formic acid and $5 \%$ acetonitrile.

\section{Sample Preparation}

502 Proteins were precipitated in 23\% TCA (Sigma-Aldrich, St. Louis, MO, Product number T-0699) 503 at $4{ }^{\circ} \mathrm{C} \mathrm{O} / \mathrm{N}$. After 30 min centrifugation at $18000 \mathrm{xg}$, protein pellets were washed 2 times with 500 504 ul ice-cold acetone. Air-dried pellets were dissolved in $8 \mathrm{M}$ urea/ $100 \mathrm{mM}$ Tris pH 8.5. Proteins 505 were reduced with $5 \mathrm{mM}$ Tris(2-carboxyethyl)phosphine hydrochloride (Sigma-Aldrich, St. Louis, $506 \mathrm{MO}$, product C4706) and alkylated with $55 \mathrm{mM}$ iodoacetamide (Sigma-Aldrich, St. Louis, MO, 507 product 111490 ). Proteins were digested for $18 \mathrm{hr}$ at $37^{\circ} \mathrm{C}$ in $2 \mathrm{M}$ urea $100 \mathrm{mM}$ Tris pH 8.5, $1 \mathrm{mM}$ $508 \mathrm{CaCl}_{2}$ with 2 ug trypsin (Promega, Madison, WI, product V5111). Digest was stopped with formic 509 acid, 5\% final concentration. Debris was removed by centrifugation, 30 min 18000xg.

\section{MudPIT Microcolumn}

511 A MudPIT microcolumn(4) was prepared by first creating a Kasil frit at one end of an 512 undeactivated $250 \square \mathrm{m}$ ID/360 $\square \mathrm{m}$ OD capillary (Agilent Technologies, Inc., Santa Clara, CA). 513 The Kasil frit was prepared by briefly dipping a 20 - $30 \mathrm{~cm}$ capillary in well-mixed 300 L Kasil 5141624 (PQ Corporation, Malvern, PA) and $100 \square \mathrm{L}$ formamide, curing at $100^{\circ} \mathrm{C}$ for $4 \mathrm{hrs}$, and cutting 
515 the frit to $\sim 2 \mathrm{~mm}$ in length. Strong cation exchange particles (SCX Luna, $5 \square \mathrm{m}$ dia., $125 \AA$ A pores,

516 Phenomenex, Torrance, CA) was packed in-house from particle slurries in methanol $2.5 \mathrm{~cm}$. An

517 additional $2.5 \mathrm{~cm}$ reversed phase particles (C18 Aqua, $3 \mu \mathrm{m}$ dia., $125 \AA$ pores, Phenomenex)

518 were then similarly packed into the capillary using the same method as SCX loading, to create a

519 biphasic column. An analytical RPLC column was generated by pulling a $100 \square \mathrm{m}$ ID/360 $\square \mathrm{m}$ OD

520 capillary (Polymicro Technologies, Inc, Phoenix, AZ) to $5 \square \mathrm{m}$ ID tip. Reversed phase particles

521 (Aqua C18, $3 \square \mathrm{m}$ dia., $125 \AA$ pores, Phenomenex, Torrance, CA) were packed directly into the

522 pulled column at 800 psi until $12 \mathrm{~cm}$ long. The MudPIT microcolumn was connected to an

523 analytical column using a zero-dead volume union (Upchurch Scientific (IDEX Health \& Science),

524 P-720-01, Oak Harbor, WA).

525

LC-MS/MS analysis was performed using an Eksigent nano lc pump and a Thermo LTQ-Orbitrap using an in-house built electrospray stage. MudPIT experiments were performed where each step corresponds to 0,20,50 and 100\% buffer $\mathrm{C}$ being run for $3 \mathrm{~min}$ at the beginning of each gradient of buffer B. Electrospray was performed directly from the analytical column by applying the ESI voltage at a tee (150 $\square \mathrm{m}$ ID, Upchurch Scientific). Electrospray directly from the LC column was done at $2.5 \mathrm{kV}$ with an inlet capillary temperature of $275^{\circ} \mathrm{C}$. Data-dependent acquisition of MS/MS spectra with the LTQ -Orbitrap were performed with the following settings: MS/MS on the 10 most intense ions per precursor scan, 1 microscan, reject charge unassigned charge state; dynamic exclusion repeat count, 1, repeat duration, 30 second; exclusion list size 200; and exclusion duration, 15 second.

\section{Data Analysis}

537 Protein and peptide identification and protein quantitation were done with Integrated Proteomics

538 Pipeline - IP2 (Integrated Proteomics Applications, Inc., San Diego, CA. 539 http://www.integratedproteomics.com/). Tandem mass spectra were extracted from raw files 540 using RawExtract 1.9.9(1) and were searched against Uniprot human database with reversed 541 sequences using ProLuCID $(2,5)$. The search space included half- and fully-tryptic peptide 542 candidates. Carbamidomethylation $(+57.02146)$ of cysteine was considered as a static 543 modification. Biotinylation of lysine (226.077598) was considered as a variable modification, 544 Peptide candidates were filtered using DTASelect, with these parameters - $\mathrm{p} 2$-y 2 --trypstat --pfp 5450.01 --extra --pl -DM 10 --DB --dm -in -m 1 -t 1 --brief --quiet $(1,3)$.

\section{Analysis of BiolD hits}


BirA*-ADAR1 and BirA*-ADAR2 experiments were analyzed separately. In both cases, peptide counts were summed to generate protein-level counts. For each biological replicate, the fold

550 change of the ADAR condition vs the no-BirA* and GFP conditions were separately calculated

551 (1 was added to all counts in order to avoid infinite fold changes). Proteins were then filtered

552 according to the following criteria: for each replicate, in order to be retained, a protein was

553 required to have a fold change $>2$ versus both the no-BirA* and GFP conditions. A second level

554 of filtering then required each protein to be retained in at least 2 out of the 3 biological

555 replicates. These retained proteins were considered hits for that condition. For heatmaps, $\log _{2}$

556 fold change for the ADAR condition was calculated by determining the median $\log _{2}$ fold change

557 versus the GFP condition across all three replicates.

558

559 Immunoprecipitation

560 FLAG-tagged proteins were immunoprecipitated from $2 \mathrm{mg}$ of HEK293T or M17 cells using anti-

561 FLAG M2 affinity gel (Sigma A2220). Lysates were prepared in NP40 buffer (see Western

562 blotting). $40 \mathrm{ul}$ of the anti-FLAG M2 affinity resin was washed three times with $1 \mathrm{ml}$ of lysis

563 buffer then added to the $2 \mathrm{mg}$ of protein extract diluted to $500 \mathrm{ul}$ in NP-40 lysis buffer and

564 incubated for 2 hours at $4^{\circ} \mathrm{C}$ with rotation. The resin was then washed 4 times for 5 minutes

565 each with $1 \mathrm{~mL} \mathrm{NP}-40$ lysis buffer at $4^{\circ} \mathrm{C}$ with rotation. For further analysis the resin was

566 resuspended with $30 \mathrm{ul}$ 2x SDS-PAGE sample buffer, then incubated at 95 degrees for 5 min to

567 elute bound proteins.

568

\section{Western Blotting}

570 Cells were lysed in NP-40 buffer (25 mM HEPES-KOH, $150 \mathrm{mM} \mathrm{KCl,} 1.5 \mathrm{mM} \mathrm{MgCl}, 0.5 \%$

571 NP40, 10\% Glycerol [pH 7.5]) supplemented with protease inhibitors (cOmplete, Mini, EDTA-

572 free Protease Inhibitor Cocktail Tablets, \# 4693159001) Lysates were clarified by spinning for

$57310 \mathrm{~min}$ at $13,000 \mathrm{rpm}$ at $4^{\circ} \mathrm{C}$, and the protein content was measured by BCA (Pierce, 23225). 10

574 ug of protein was separated by SDS-PAGE, transferred onto nitrocellulose membrane, and

575 blotted according to standard protocols. Chemiluminescence was imaged using a BioRad

576 ChemiDoc imaging system.

\section{$578 \quad$ Antibodies}

579 ILF2: Bethyl laboratories NF45 Antibody, cat: A303-147A (1:1000)

580 ILF3: Bethyl laboratories NF90 Antibody, cat: A303-651A (1:1000)

581 ZFR: Abcam Anti-ZFR antibody ab90865 (1:500) 
ADAR2: Genetex ADAR2 antibody cat: GTX114237(1:500)

FLAG: Sigma Monoclonal ANTI-FLAG® M2-Peroxidase (HRP) (1:5000)

\section{mmPCR-seq of samples}

588 The mmPCR-seq was performed as described in (Zhang et al., 2014). Briefly, total RNA is 589 extracted from cells using a Qiagen Micro or Mini RNeasy kit (Qiagen cat:74004 or 74104) and 590 reverse transcribed using iScript Advanced reverse transcriptase (Bio-Rad). The cDNAs were 591 purified using Ampure XP Beads (Beckman Coulter), with an elution volume of 10 ul. $250 \mathrm{ng}$

592 of cDNA was used in a preamplification reaction and amplified cDNA was purified using 593 Ampure XP beads and eluted in $10 \mathrm{ul}$. $50 \mathrm{ng}$ of pre-amplified cDNA was loaded into each 594 well of an Access Array microfluidic chip (Fluidigm). The PCR reactions were performed on 595 the Access Array System (Fluidigm) using KAPA2G 5X Fast Multiplex PCR Mix (Kapa 596 Biosystems). Barcodes were added in a second round of PCR using Phusion DNA polymerase (NEB cat: M0531S). Samples were sequenced with 76 base-pair paired-end reads using an Illumina NextSeq (Illumina, San Diego, CA).

\section{Analysis of mmPCR-seq and RNA-seq} mmPCR-seq and RNA-seq reads were mapped to the human genome (hg19) using STAR (Dobin et al., 2013) version 2.4.2a using the parameters (--outFilterMultimapNmax 20 -outFilterMismatchNmax 999 --outFilterMismatchNoverReadLmax 0.1 --alignIntronMin 20 -alignIntronMax 1000000 --alignMatesGapMax 1000000 --alignSJoverhangMin 8 -alignSJDBoverhangMin 1 --sjdbScore 1 --twopassMode Basic). For editing analysis we used the Samtools (version 0.1.16) (Li et al., 2009) mpileup command to count A and G counts at known editing sites (Tan et al., 2017), RADAR version 2 (Ramaswami and Li, 2014). For RNA-seq only bases with quality scores $>20$ were used. For both RNA-seq and mmPCR-seq, combined A and $G$ counts from two replicates of each condition were compared to down sampled GFP overexpression reads using Fisher's exact test with a Benjimini-Hochberg multiple hypothesis

611 testing correction in R (Benjamini and Hochberg, 1995).

613 For both RNA-seq and mmPCR-seq analysis, any variability between replicates could arise for a 614 number of reasons, e.g. low expression or inefficient primer-based amplification, so for all 
615 subsequent analyses we only assessed sites with high coverage (50X in mmPCR-seq and 20X

616 in RNA-seq) that were $<10 \%$ different between replicates (Figure S6A, B).

618 For gene expression analysis, FPKMs were calculated using RSEM 1.2.21 (Li and Dewey,

619 2011). To compare global expression differences of cell-type-specific BiolD hits in M17 and

620 HeLa cells, we used Wilcoxon matched-pairs signed ranked test performed by Graphpad

621 PRISM 7.

622

\section{RNA-seq library preparation}

624 Total RNA was extracted from cells using Qiagen Micro or Mini RNeasy kit (Qiagen cat:74004

625 or 74104). rRNA was depleted from total RNA following RNase H-based protocols adopted from

626 (Adiconis et al., 2013; Morlan et al., 2012). We mixed approximately 250 ng of RNA with 61.54

627 pmoles of pooled DNA oligos designed antisense to rRNA (gift from J. Salzman lab at Stanford)

628 in a $5 u$ reaction with $1 \mathrm{ul}$ of $5 \mathrm{X} \mathrm{RNase} \mathrm{H}(-) \mathrm{Mg}$ buffer $(500 \mathrm{mM}$ Tris- $\mathrm{HCl} \mathrm{pH} \mathrm{7.4,1} \mathrm{M} \mathrm{NaCl})$ and

6290.25 ul 1mM EDTA. We annealed rRNA antisense oligos to total RNA samples for 2 minutes at

$63095^{\circ} \mathrm{C}$, slowly reduced the temperature to $45^{\circ} \mathrm{C}$ and then added $5 \mathrm{ul}$ of RNase $\mathrm{H}$ mix (1.7 ul

631 water, 1 ul 5X RNase H(-)Mg buffer, 0.2 ul $1 \mathrm{M} \mathrm{MgCl}_{2}, 0.1$ ul RiboLock RNase Inhibitor (40 U/ $\left.\mu \mathrm{L}\right)$

632 (ThermoFisher EO038), 2 ul of Hybridase Thermostable RNase H (Epicenter, Madison, WI:

633 Lucigen $\mathrm{H} 39500$ ) to make $10 \mathrm{ul}$ total and incubated for 30 minutes at $45^{\circ} \mathrm{C}$. rRNA-depleted RNA

634 was then purified using 2.2X reaction volume of Agencourt RNAClean XP beads (Beckman

635 Coulter: A63987), treated with TURBO DNase (Invitrogen: AM1907), and then purified with

636 RNAClean XP beads again. rRNA-depleted RNA was used as input to the KAPA HyperPrep

637 RNA-seq Kit (Kapa Biosystems: KK8540). All libraries were sequenced with 76 base-pair

638 paired-end reads using an Illumina NextSeq (Illumina, San Diego, CA).

639

640 Sanger Sequencing of RNA levels

641 To determine editing levels at PAICS and GRIA2 editing sites, RNA was extracted from M17

642 cells expressing BirA*-GFP, BirA*-ADAR1, and BirA*-ADAR2 using Zymo Quick RNA kit. RNA

643 was treated with TURBO DNase and then cDNA was synthesized using Bio-Rad iScript

644 Advanced cDNA synthesis kit. PCR was performed using the following to amplify around the

645 editing site in each gene, PAICS FWD: TCAATCCACCCTTTTCCAAG, REV:

646 TGATAAAAACGTGGGCCTTC and GRIA2 FWD: CAGCAGATTTAGCCCCTACG REV:

647 AGATGAGATGTGTGCCAACG) with NEB Phusion for 40 cycles, and amplicons were gel 
648 purified with Qiagen QIAquick Gel Extraction kit (Cat 28115) and sent for Sanger sequencing.

649 Two replicates were performed for each group of cells.

650

\section{ENCODE data analysis}

652 We downloaded the mapped BAM files (HG38 version) of RNA-seq data generated following

653 RBP knockdown or control shRNA transfection from the ENCODE data portal

654 (encodeproject.org). For the RNA-seq data, we used the same pipeline as described in the

655 Analysis of mmPCR-seq and RNA-seq section to quantify editing levels. Similarly, as reported

656 (Quinones-Valdez et al., 2019), we also found batch effects in the editing level measurements

657 from the RNA-seq data. However, in this paper we only performed editing level comparison

658 between RBP knockdown and the matched control shRNA transfection within the same batch,

659 so it was not necessary to remove the batch effects that only affected editing level

660 measurements of samples from different batches. When the accumulative editing level

661 differences was calculated and compared between different RBPs in Fig. 2E, the influence of

662 batch effects was taken into consideration by normalizing the CDED to [0,1].

664 To determine where the RBPs bind on the RNA, we analyzed the eCLIP-seq data from

665 ENCODE. We downloaded the BED files (HG38 version) of the called CLIP peaks for each

666 eCLIP-seq data and used shifted z-score method to test how the strength of association

667 between the RBP binding and the editing sites would change if the peak was shifted from its

668 original position (Gel et al., 2016). The z-scores were calculated as the distance between the

669 expected binding value and the observed one, measured in standard deviations. And we shifted

670 the peaks 50bp stepwise within the $1.2 \mathrm{~kb}$ up- and down-stream windows of the editing site to

671 obtain the corresponding z-score values.

672

\section{Accession Numbers}

674 The high-throughput sequencing data utilized in this work, including the RNA-seq and mmPCR-

675 seq libraries, have been deposited in the Gene Expression Omnibus (GEO) database,

676 accession number GSE130771.

677

\section{ACKNOWLEDGEMENTS}

679 We thank Adam Freund, and all members of the Li Lab especially Tao Sun for useful

680 discussions and comments on this paper. We would also like to thank Julia Salzman for her

681 generous donation of the oligos used for rRNA depletion in the RNA-seq library preparation. 
682 Funding sources: American Heart Association Postdoctoral grant 16POST27700036 (to E.C.F), 683 National Center for Research Resources 5P41RR011823 (to JJM and JRY), NIH R01-

684 GM102484 (to J.B.L.), R01-GM124215 (to J.B.L.), R01-MH115080 (to J.B.L.)

685 


\section{REFERENCES}

A Large-Scale Binding and Functional Map of Human RNA Binding Proteins | bioRxiv [WWW Document], n.d. URL https://www.biorxiv.org/content/10.1101/179648v2 (accessed 3.28.19).

Aktaş, T., Avşar Ilık, I., Maticzka, D., Bhardwaj, V., Pessoa Rodrigues, C., Mittler, G., Manke, T., Backofen, R., Akhtar, A., 2017. DHX9 suppresses RNA processing defects originating from the Alu invasion of the human genome. Nature 544, 115-119. https://doi.org/10.1038/nature21715

Anantharaman, A., Jadaliha, M., Tripathi, V., Nakagawa, S., Hirose, T., Jantsch, M.F., Prasanth, S.G., Prasanth, K.V., 2016. Paraspeckles modulate the intranuclear distribution of paraspeckle-associated Ctn RNA. Sci. Rep. 6. https://doi.org/10.1038/srep34043

Athanasiadis, A., Rich, A., Maas, S., 2004. Widespread A-to-I RNA editing of Alu-containing mRNAs in the human transcriptome. PLoS Biol. 2, e391. https://doi.org/10.1371/journal.pbio.0020391

Bazak, L., Levanon, E.Y., Eisenberg, E., 2014. Genome-wide analysis of Alu editability. Nucleic Acids Res. 42, 6876-6884. https://doi.org/10.1093/nar/gku414

Behm, M., Wahlstedt, H., Widmark, A., Eriksson, M., Öhman, M., 2017. Accumulation of nuclear ADAR2 regulates adenosine-to-inosine RNA editing during neuronal development. J. Cell Sci. 130, 745-753. https://doi.org/10.1242/jcs.200055

Bhate, A., Sun, T., Li, J.B., 2019. ADAR1: A New Target for Immuno-oncology Therapy. Mol. Cell 73, 866-868. https://doi.org/10.1016/j.molcel.2019.02.021

Binothman, N., Hachim, I.Y., Lebrun, J.-J., Ali, S., 2017. CPSF6 is a Clinically Relevant Breast Cancer Vulnerability Target: Role of CPSF6 in Breast Cancer. EBioMedicine 21, 65-78. https://doi.org/10.1016/j.ebiom.2017.06.023

Blango, M.G., Bass, B.L., 2016. Identification of the long, edited dsRNAome of LPS-stimulated immune cells. Genome Res. 26, 852-862. https://doi.org/10.1101/gr.203992.116

Blow, M., Futreal, P.A., Wooster, R., Stratton, M.R., 2004. A survey of RNA editing in human brain. Genome Res. 14, 2379-2387. https://doi.org/10.1101/gr.2951204

Burns, C.M., Chu, H., Rueter, S.M., Hutchinson, L.K., Canton, H., Sanders-Bush, E., Emeson, R.B., 1997. Regulation of serotonin-2C receptor G-protein coupling by RNA editing. Nature 387, 303-308. https://doi.org/10.1038/387303a0

Castello, A., Fischer, B., Frese, C.K., Horos, R., Alleaume, A.-M., Foehr, S., Curk, T., Krijgsveld, J., Hentze, M.W., 2016. Comprehensive Identification of RNA-Binding Domains in Human Cells. Mol. Cell 63, 696-710. https://doi.org/10.1016/j.molcel.2016.06.029

Chu, L., Su, M.Y., Maggi, L.B., Lu, L., Mullins, C., Crosby, S., Huang, G., Chng, W.J., Vij, R., Tomasson, M.H., 2012. Multiple myeloma-associated chromosomal translocation activates orphan snoRNA ACA11 to suppress oxidative stress. J. Clin. Invest. 122, 2793-2806. https://doi.org/10.1172/JCl63051

Dobin, A., Davis, C.A., Schlesinger, F., Drenkow, J., Zaleski, C., Jha, S., Batut, P., Chaisson, M., Gingeras, T.R., 2013. STAR: ultrafast universal RNA-seq aligner. Bioinforma. Oxf. Engl. 29, 15-21. https://doi.org/10.1093/bioinformatics/bts635

Eisenberg, E., Levanon, E.Y., 2018. A-to-I RNA editing - immune protector and transcriptome diversifier. Nat. Rev. Genet. 19, 473-490. https://doi.org/10.1038/s41576-018-0006-1

Gannon, H.S., Zou, T., Kiessling, M.K., Gao, G.F., Cai, D., Choi, P.S., Ivan, A.P., Buchumenski, I., Berger, A.C., Goldstein, J.T., Cherniack, A.D., Vazquez, F., Tsherniak, A., Levanon, E.Y., Hahn, W.C., Meyerson, M., 2018. Identification of ADAR1 adenosine deaminase dependency in a subset of cancer cells. Nat. Commun. 9, 5450. https://doi.org/10.1038/s41467-018-07824-4 
Garncarz, W., Tariq, A., Handl, C., Pusch, O., Jantsch, M.F., 2013. A high-throughput screen to identify enhancers of ADAR-mediated RNA-editing. RNA Biol. 10, 192-204. https://doi.org/10.4161/rna.23208

Gel, B., Díez-Villanueva, A., Serra, E., Buschbeck, M., Peinado, M.A., Malinverni, R., 2016. regioneR: an $\mathrm{R} /$ Bioconductor package for the association analysis of genomic regions based on permutation tests. Bioinforma. Oxf. Engl. 32, 289-291. https://doi.org/10.1093/bioinformatics/btv562

Hirose, T., Virnicchi, G., Tanigawa, A., Naganuma, T., Li, R., Kimura, H., Yokoi, T., Nakagawa, S., Bénard, M., Fox, A.H., Pierron, G., 2014. NEAT1 long noncoding RNA regulates transcription via protein sequestration within subnuclear bodies. Mol. Biol. Cell 25, 169183. https://doi.org/10.1091/mbc.E13-09-0558

Huang, H., Kapeli, K., Jin, W., Wong, Y.P., Arumugam, T.V., Koh, J.H., Srimasorn, S., Mallilankaraman, K., Chua, J.J.E., Yeo, G.W., Soong, T.W., 2018. Tissue-selective restriction of RNA editing of CaV1.3 by splicing factor SRSF9. Nucleic Acids Res. 46, 7323-7338. https://doi.org/10.1093/nar/gky348

Ishizuka, J.J., Manguso, R.T., Cheruiyot, C.K., Bi, K., Panda, A., Iracheta-Vellve, A., Miller, B.C., Du, P.P., Yates, K.B., Dubrot, J., Buchumenski, I., Comstock, D.E., Brown, F.D., Ayer, A., Kohnle, I.C., Pope, H.W., Zimmer, M.D., Sen, D.R., Lane-Reticker, S.K., Robitschek, E.J., Griffin, G.K., Collins, N.B., Long, A.H., Doench, J.G., Kozono, D., Levanon, E.Y., Haining, W.N., 2019. Loss of ADAR1 in tumours overcomes resistance to immune checkpoint blockade. Nature 565, 43-48. https://doi.org/10.1038/s41586-0180768-9

Kim, D.I., Birendra, K.C., Zhu, W., Motamedchaboki, K., Doye, V., Roux, K.J., 2014. Probing nuclear pore complex architecture with proximity-dependent biotinylation. Proc. Natl. Acad. Sci. U. S. A. 111, E2453-2461. https://doi.org/10.1073/pnas.1406459111

Kwon, K., Streaker, E.D., Ruparelia, S., Beckett, D., 2000. Multiple disordered loops function in corepressor-induced dimerization of the biotin repressor. J. Mol. Biol. 304, 821-833. https://doi.org/10.1006/jmbi.2000.4249

Levanon, E.Y., Eisenberg, E., Yelin, R., Nemzer, S., Hallegger, M., Shemesh, R., Fligelman, Z.Y., Shoshan, A., Pollock, S.R., Sztybel, D., Olshansky, M., Rechavi, G., Jantsch, M.F., 2004. Systematic identification of abundant A-to-I editing sites in the human transcriptome. Nat. Biotechnol. 22, 1001-1005. https://doi.org/10.1038/nbt996

Li, B., Dewey, C.N., 2011. RSEM: accurate transcript quantification from RNA-Seq data with or without a reference genome. BMC Bioinformatics 12, 323. https://doi.org/10.1186/14712105-12-323

Li, H., Handsaker, B., Wysoker, A., Fennell, T., Ruan, J., Homer, N., Marth, G., Abecasis, G., Durbin, R., 1000 Genome Project Data Processing Subgroup, 2009. The Sequence Alignment/Map format and SAMtools. Bioinforma. Oxf. Engl. 25, 2078-2079. https://doi.org/10.1093/bioinformatics/btp352

Li, X., Liu, C.-X., Xue, W., Zhang, Y., Jiang, S., Yin, Q.-F., Wei, J., Yao, R.-W., Yang, L., Chen, L.-L., 2017. Coordinated circRNA Biogenesis and Function with NF90/NF110 in Viral Infection. Mol. Cell 67, 214-227.e7. https://doi.org/10.1016/j.molcel.2017.05.023

Liddicoat, B.J., Piskol, R., Chalk, A.M., Ramaswami, G., Higuchi, M., Hartner, J.C., Li, J.B., Seeburg, P.H., Walkley, C.R., 2015. RNA editing by ADAR1 prevents MDA5 sensing of endogenous dsRNA as nonself. Science 349, 1115-1120. https://doi.org/10.1126/science.aac7049

Liu, H., Golji, J., Brodeur, L.K., Chung, F.S., Chen, J.T., deBeaumont, R.S., Bullock, C.P., Jones, M.D., Kerr, G., Li, L., Rakiec, D.P., Schlabach, M.R., Sovath, S., Growney, J.D., Pagliarini, R.A., Ruddy, D.A., Maclsaac, K.D., Korn, J.M., McDonald, E.R., 2019. Tumorderived IFN triggers chronic pathway agonism and sensitivity to ADAR loss. Nat. Med. 25, 95. https://doi.org/10.1038/s41591-018-0302-5 
785

786

787

788

789

790

791

792

793

794

795

796

797

798

799

800

801

802

803

804

805

806

807

808

809

810

811

812

813

814

815

816

817

818

819

820

821

822

823

824

825

826

827

828

829

830

831

832

833

834

835
Mannion, N.M., Greenwood, S.M., Young, R., Cox, S., Brindle, J., Read, D., Nellåker, C., Vesely, C., Ponting, C.P., McLaughlin, P.J., Jantsch, M.F., Dorin, J., Adams, I.R., Scadden, A.D.J., Ohman, M., Keegan, L.P., O'Connell, M.A., 2014. The RNA-editing enzyme ADAR1 controls innate immune responses to RNA. Cell Rep. 9, 1482-1494. https://doi.org/10.1016/j.celrep.2014.10.041

Marcucci, R., Brindle, J., Paro, S., Casadio, A., Hempel, S., Morrice, N., Bisso, A., Keegan, L.P., Del Sal, G., O'Connell, M.A., 2011. Pin1 and WWP2 regulate GluR2 Q/R site RNA editing by ADAR2 with opposing effects. EMBO J. 30, 4211-4222. https://doi.org/10.1038/emboj.2011.303

Nemlich, Y., Baruch, E.N., Besser, M.J., Shoshan, E., Bar-Eli, M., Anafi, L., Barshack, I., Schachter, J., Ortenberg, R., Markel, G., 2018. ADAR1-mediated regulation of melanoma invasion. Nat. Commun. 9, 2154. https://doi.org/10.1038/s41467-018-046002

Nie, Y., Ding, L., Kao, P.N., Braun, R., Yang, J.-H., 2005. ADAR1 interacts with NF90 through double-stranded RNA and regulates NF90-mediated gene expression independently of RNA editing. Mol. Cell. Biol. 25, 6956-6963. https://doi.org/10.1128/MCB.25.16.69566963.2005

Nishikura, K., 2016. A-to-I editing of coding and non-coding RNAs by ADARs. Nat. Rev. Mol. Cell Biol. 17, 83-96. https://doi.org/10.1038/nrm.2015.4

Nishikura, K., 2010. Functions and regulation of RNA editing by ADAR deaminases. Annu. Rev. Biochem. 79, 321-349. https://doi.org/10.1146/annurev-biochem-060208-105251

Orecchini, E., Frassinelli, L., Michienzi, A., 2017. Restricting retrotransposons: ADAR1 is another guardian of the human genome. RNA Biol. 14, 1485-1491. https://doi.org/10.1080/15476286.2017.1341033

Pestal, K., Funk, C.C., Snyder, J.M., Price, N.D., Treuting, P.M., Stetson, D.B., 2015. Isoforms of RNA-Editing Enzyme ADAR1 Independently Control Nucleic Acid Sensor MDA5Driven Autoimmunity and Multi-organ Development. Immunity 43, 933-944. https://doi.org/10.1016/j.immuni.2015.11.001

Quinones-Valdez, G., Tran, S.S., Jun, H.-I., Bahn, J.H., Yang, E.-W., Zhan, L., Brümmer, A., Wei, X., Van Nostrand, E.L., Pratt, G.A., Yeo, G.W., Graveley, B.R., Xiao, X., 2019. Regulation of RNA editing by RNA-binding proteins in human cells. Commun. Biol. 2. https://doi.org/10.1038/s42003-018-0271-8

Rice, G.I., Kasher, P.R., Forte, G.M.A., Mannion, N.M., Greenwood, S.M., Szynkiewicz, M., Dickerson, J.E., Bhaskar, S.S., Zampini, M., Briggs, T.A., Jenkinson, E.M., Bacino, C.A., Battini, R., Bertini, E., Brogan, P.A., Brueton, L.A., Carpanelli, M., De Laet, C., de Lonlay, P., del Toro, M., Desguerre, I., Fazzi, E., Garcia-Cazorla, A., Heiberg, A., Kawaguchi, M., Kumar, R., Lin, J.-P.S.-M., Lourenco, C.M., Male, A.M., Marques, W., Mignot, C., Olivieri, I., Orcesi, S., Prabhakar, P., Rasmussen, M., Robinson, R.A., Rozenberg, F., Schmidt, J.L., Steindl, K., Tan, T.Y., van der Merwe, W.G., Vanderver, A., Vassallo, G., Wakeling, E.L., Wassmer, E., Whittaker, E., Livingston, J.H., Lebon, P., Suzuki, T., McLaughlin, P.J., Keegan, L.P., O'Connell, M.A., Lovell, S.C., Crow, Y.J., 2012. Mutations in ADAR1 cause Aicardi-Goutières syndrome associated with a type I interferon signature. Nat. Genet. 44, 1243-1248. https://doi.org/10.1038/ng.2414

Roux, K.J., Kim, D.I., Raida, M., Burke, B., 2012. A promiscuous biotin ligase fusion protein identifies proximal and interacting proteins in mammalian cells. J. Cell Biol. 196, 801810. https://doi.org/10.1083/jcb.201112098

Rueter, S.M., Dawson, T.R., Emeson, R.B., 1999. Regulation of alternative splicing by RNA editing. Nature 399, 75-80. https://doi.org/10.1038/19992

Sapiro, A.L., Freund, E.C., Restrepo, L., Qiao, H.-H., Bhate, A., Ni, J.-Q., Mosca, T.J., Li, J.B., n.d. Zinc finger RNA binding protein Zn72D regulates ADAR-mediated RNA editing in neurons. BioRxiv, http://biorxiv.org/cgi/content/short/631986v1 
836

837

838

839

840

841

842

843

844

845

846

847

848

849

850

851

852

853

854

855

856

857

858

859

860

861

862

863

864

865

866

867

868

869

870

871

872

873

874

875

876

877

878

879

880

881

882

883

884

885

886
Sapiro, A.L., Shmueli, A., Henry, G.L., Li, Q., Shalit, T., Yaron, O., Paas, Y., Li, J.B., ShohatOphir, G., 2019. Illuminating spatial A-to-I RNA editing signatures within the Drosophila brain. Proc. Natl. Acad. Sci. 116, 2318-2327. https://doi.org/10.1073/pnas.1811768116

Shanmugam, R., Zhang, F., Srinivasan, H., Charles Richard, J.L., Liu, K.I., Zhang, X., Woo, C.W.A., Chua, Z.H.M., Buschdorf, J.P., Meaney, M.J., Tan, M.H., 2018. SRSF9 selectively represses ADAR2-mediated editing of brain-specific sites in primates. Nucleic Acids Res. 46, 7379-7395. https://doi.org/10.1093/nar/gky615

Slotkin, W., Nishikura, K., 2013. Adenosine-to-inosine RNA editing and human disease. Genome Med. 5, 105. https://doi.org/10.1186/gm508

Stellos, K., Gatsiou, A., Stamatelopoulos, K., Perisic Matic, L., John, D., Lunella, F.F., Jaé, N., Rossbach, O., Amrhein, C., Sigala, F., Boon, R.A., Fürtig, B., Manavski, Y., You, X., Uchida, S., Keller, T., Boeckel, J.-N., Franco-Cereceda, A., Maegdefessel, L., Chen, W., Schwalbe, H., Bindereif, A., Eriksson, P., Hedin, U., Zeiher, A.M., Dimmeler, S., 2016. Adenosine-to-inosine RNA editing controls cathepsin $\mathrm{S}$ expression in atherosclerosis by enabling HuR-mediated post-transcriptional regulation. Nat. Med. 22, 1140-1150. https://doi.org/10.1038/nm.4172

Sundararaman, B., Zhan, L., Blue, S.M., Stanton, R., Elkins, K., Olson, S., Wei, X., Van Nostrand, E.L., Pratt, G.A., Huelga, S.C., Smalec, B.M., Wang, X., Hong, E.L., Davidson, J.M., Lécuyer, E., Graveley, B.R., Yeo, G.W., 2016. Resources for the Comprehensive Discovery of Functional RNA Elements. Mol. Cell 61, 903-913. https://doi.org/10.1016/j.molcel.2016.02.012

Tan, M.H., Li, Q., Shanmugam, R., Piskol, R., Kohler, J., Young, A.N., Liu, K.I., Zhang, R., Ramaswami, G., Ariyoshi, K., Gupte, A., Keegan, L.P., George, C.X., Ramu, A., Huang, N., Pollina, E.A., Leeman, D.S., Rustighi, A., Goh, Y.P.S., GTEx Consortium, Laboratory, Data Analysis \&Coordinating Center (LDACC)—Analysis Working Group, Statistical Methods groups-Analysis Working Group, Enhancing GTEx (eGTEx) groups, $\mathrm{NIH}$ Common Fund, NIH/NCI, NIH/NHGRI, NIH/NIMH, NIH/NIDA, Biospecimen Collection Source Site-NDRI, Biospecimen Collection Source Site-RPCI, Biospecimen Core Resource-VARI, Brain Bank Repository-University of Miami Brain Endowment Bank, Leidos Biomedical-Project Management, ELSI Study, Genome Browser Data Integration \&Visualization-EBI, Genome Browser Data Integration \&Visualization-UCSC Genomics Institute, University of California Santa Cruz, Chawla, A., Del Sal, G., Peltz, G., Brunet, A., Conrad, D.F., Samuel, C.E., O’Connell, M.A., Walkley, C.R., Nishikura, K., Li, J.B., 2017. Dynamic landscape and regulation of RNA editing in mammals. Nature 550, 249-254. https://doi.org/10.1038/nature24041

Tariq, A., Garncarz, W., Handl, C., Balik, A., Pusch, O., Jantsch, M.F., 2013. RNA-interacting proteins act as site-specific repressors of ADAR2-mediated RNA editing and fluctuate upon neuronal stimulation. Nucleic Acids Res. 41, 2581-2593. https://doi.org/10.1093/nar/gks1353

Tran, S.S., Jun, H.-I., Bahn, J.H., Azghadi, A., Ramaswami, G., Nostrand, E.L.V., Nguyen, T.B., Hsiao, Y.-H.E., Lee, C., Pratt, G.A., Martínez-Cerdeño, V., Hagerman, R.J., Yeo, G.W., Geschwind, D.H., Xiao, X., 2019. Widespread RNA editing dysregulation in brains from autistic individuals. Nat. Neurosci. 22, 25. https://doi.org/10.1038/s41593-018-0287-x

Wahlstedt, H., Daniel, C., Ensterö, M., Ohman, M., 2009. Large-scale mRNA sequencing determines global regulation of RNA editing during brain development. Genome Res. 19, 978-986. https://doi.org/10.1101/gr.089409.108

Walkley, C.R., Li, J.B., 2017. Rewriting the transcriptome: adenosine-to-inosine RNA editing by ADARs. Genome Biol. 18, 205. https://doi.org/10.1186/s13059-017-1347-3

Wolkowicz, U.M., Cook, A.G., 2012. NF45 dimerizes with NF90, Zfr and SPNR via a conserved domain that has a nucleotidyltransferase fold. Nucleic Acids Res. 40, 9356-9368. https://doi.org/10.1093/nar/gks696 
Yang, B., Hu, P., Lin, X., Han, W., Zhu, L., Tan, X., Ye, F., Wang, G., Wu, F., Yin, B., Bao, Z., Jiang, T., Yuan, J., Qiang, B., Peng, X., 2015. PTBP1 induces ADAR1 p110 isoform expression through IRES-like dependent translation control and influences cell proliferation in gliomas. Cell. Mol. Life Sci. CMLS 72, 4383-4397. https://doi.org/10.1007/s00018-015-1938-7

Zhang, R., Li, X., Ramaswami, G., Smith, K.S., Turecki, G., Montgomery, S.B., Li, J.B., 2014. Quantifying RNA allelic ratios by microfluidics-based multiplex PCR and deep sequencing. Nat. Methods 11, 51-54. https://doi.org/10.1038/nmeth.2736 
A

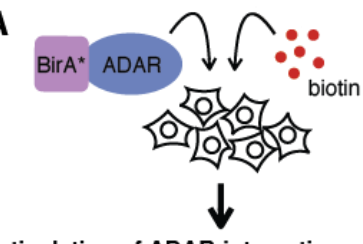

biotinylation of ADAR-interacting proteins

proximal

proteins

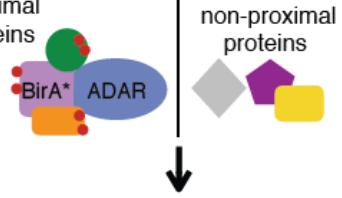

streptavidin IP

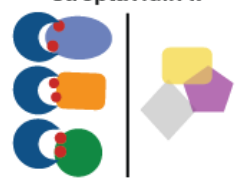

mass spec identifies proximal proteins

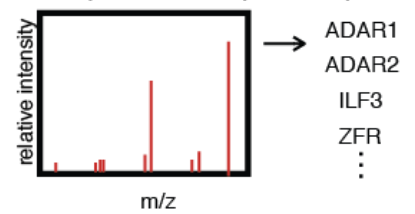

B

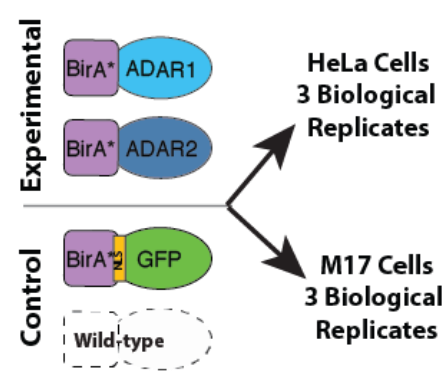

C

Calculate a fold change over the GFP and the no BirA* control for each biological replicate

Require proteins to be enriched 2X over the GFP and no BirA* controls

Require the above enrichment in at least 2 of 3 replicates

Calculate a median fold change over GFP

D

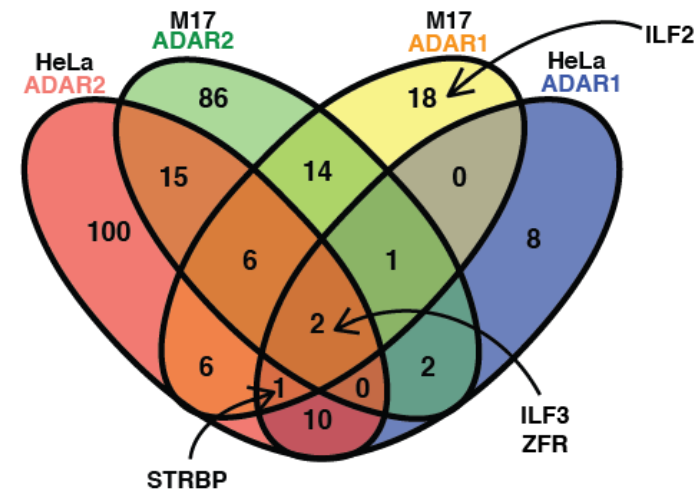

Figure 1. BiolD in human cells identifies known and novel regulators of ADAR1 and ADAR2 A. Schematic depicting BiolD protocol for ADAR. A fusion of ADAR1 or ADAR2 with the BirA* enzyme was expressed in cells and the media was supplemented with exogenous Biotin, allowing for the fusion protein to biotinylate proximal proteins. A Streptavidin IP was then performed to isolate biotinylated proteins. The eluted proteins were then identified by mass spectrometry. B. Schematic of BiolD experimental conditions. The experiment was performed with two different BiolD fusion proteins, BirA*ADAR1 and BirA*-ADAR2, and two negative controls, BirA*-GFP and untransfected cells with no BirA expressed. Each fusion protein and control was assayed in HeLa and M17 cells for a total of eight experimental conditions, performed in triplicate. C. Pipeline depicting the analysis performed to determine and rank BioID hits. A series of filters were applied using each control condition to remove false positives. Median fold change over the GFP condition was used to rank the hits. D. Venn diagram depicting the hits from each BiolD experiment. The hits from each BiolD are indicated by differently colored ovals (pink: HeLa ADAR2, green: M17 ADAR2, Yellow: M17 ADAR1, Blue: HeLa ADAR1). The numbers indicate the number of hits that overlap between each condition and the proteins denoted in bold are the BiolD hits further characterized in this study. 
A
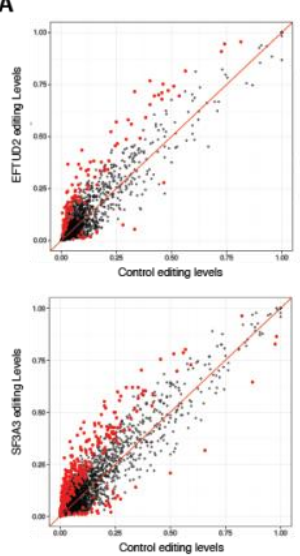

B

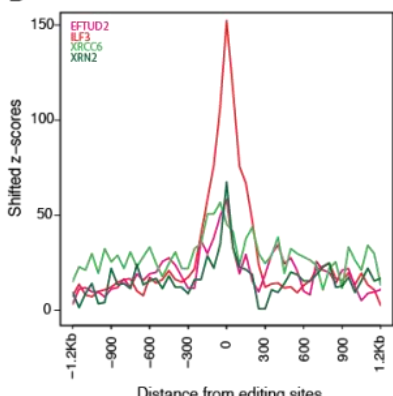

0 p-value $<0.05$
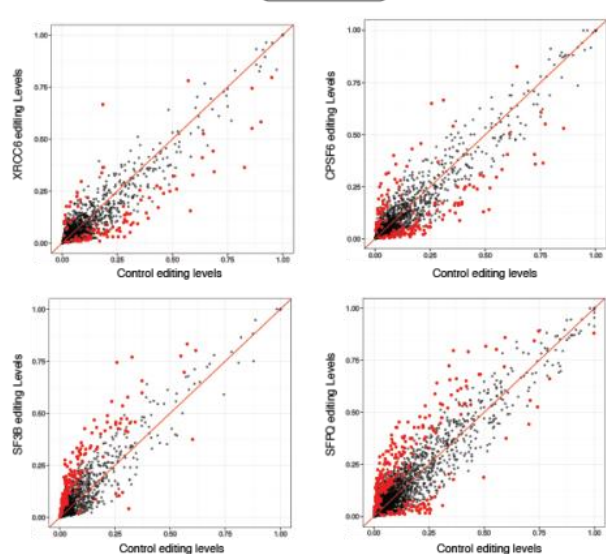
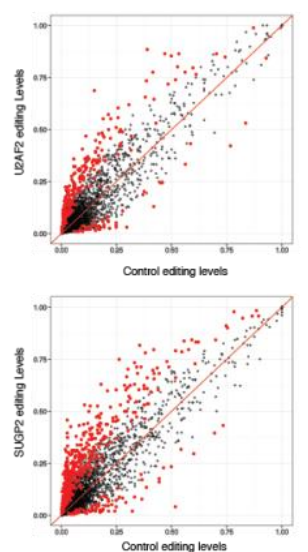

C

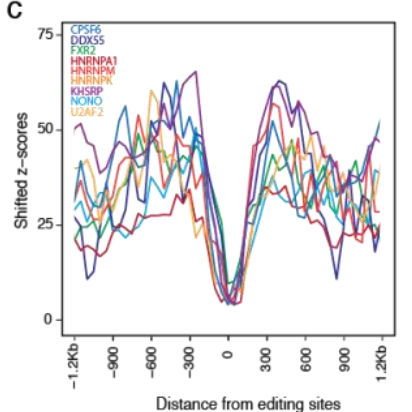

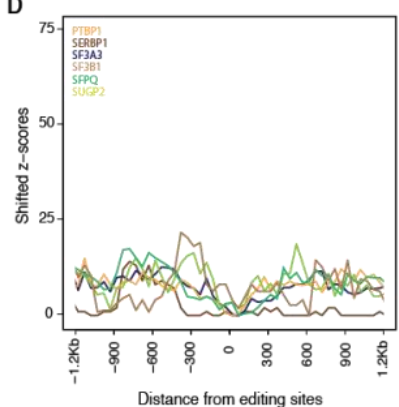

E

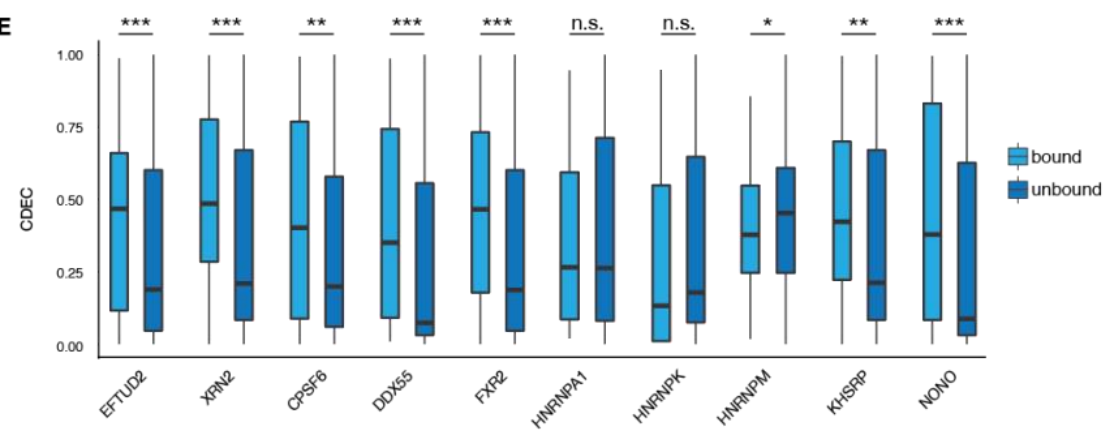

Figure 2. RNA-seq and eCLIP-seq analyses identify regulatory RNA-binding proteins of RNA editing.

A. Scatter plots of pairwise comparison of editing levels between knockdown and control RNA-seq of 8 top regulatory RBPs. Red dots, Fisher's exact test $p$-value $<0.05$. B-D. Z-scores showing the observed RBP binding strength over the expected one, measured in standard deviations (see Methods section for details). B. RBPs that bind directly to editing sites in eCLIP-seq. C. RBPs that bind near-by editing sites (non-overlapping) in eCLIP-seq. D. RBPs that show no enrichment of binding near editing sites in eCLIPseq. E. Comparison of editing profile differences in knockdowns between sites bound by RBPs (light blue) and the ones unbound (dark blue). CDED: cumulative distribution of editing level deviation, quantifies the accumulative editing level difference from the mean between controls and knockdowns (Methods). 
A
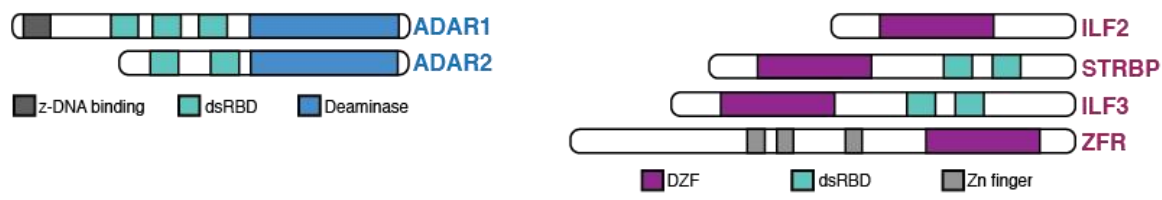

B

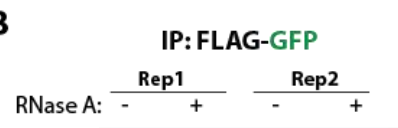

IP: FLAG-ADAR1

C $\frac{\text { Rep1 }}{-}+\frac{\text { Rep2 }}{+}$

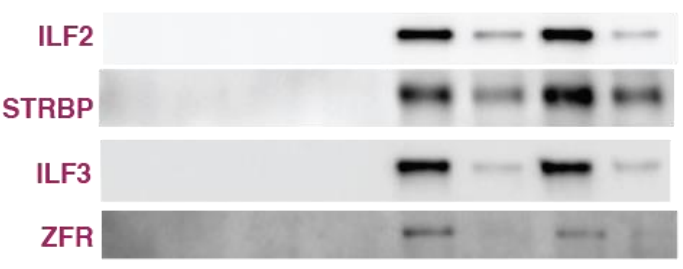

D
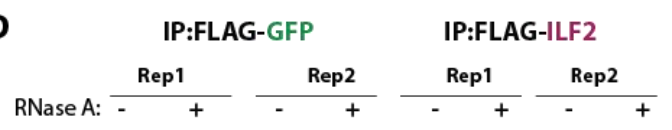

\section{E}
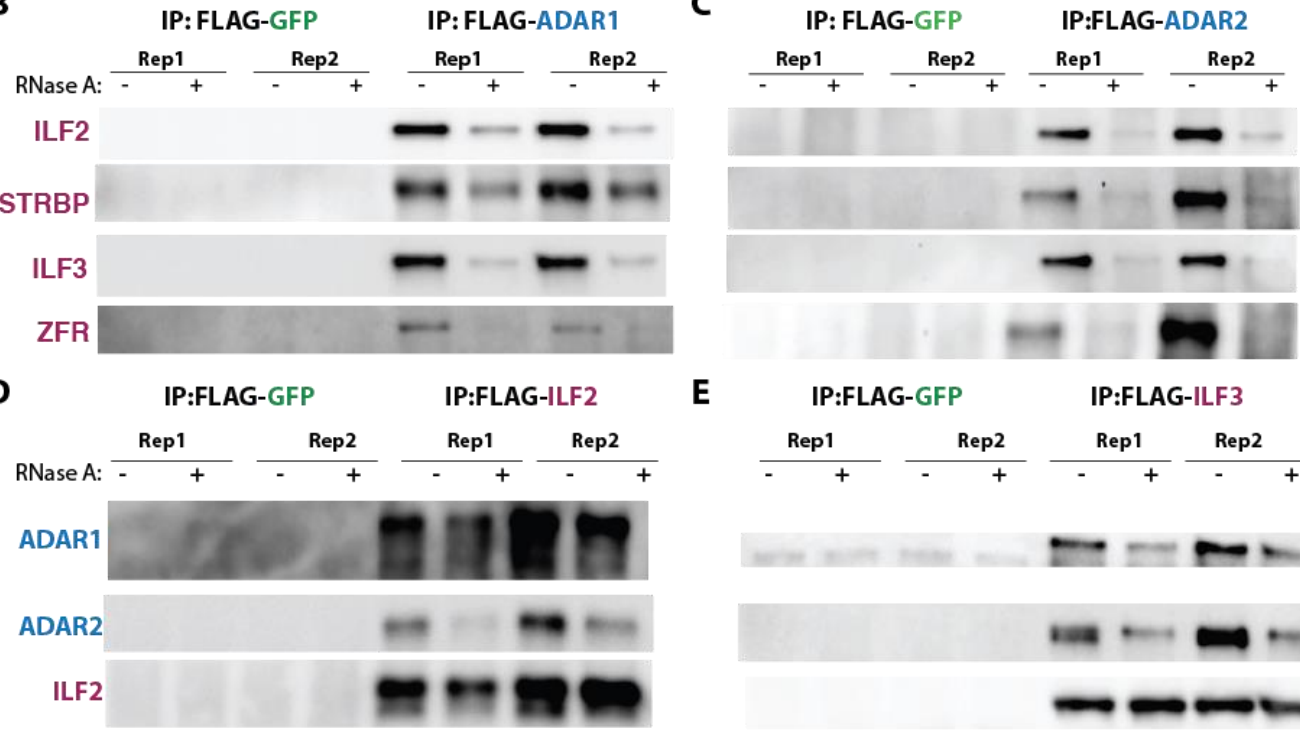

\section{F}

IP:FLAG-GFP

IP:FLAG-ZFR

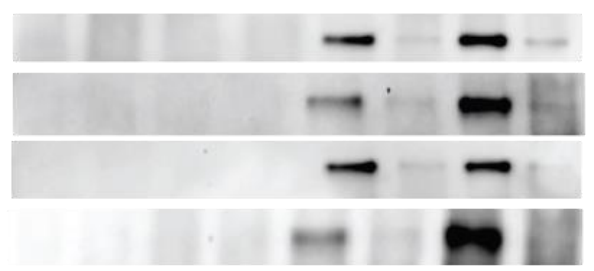

\section{ADAR1}

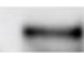

\section{ADAR2}

ILF2
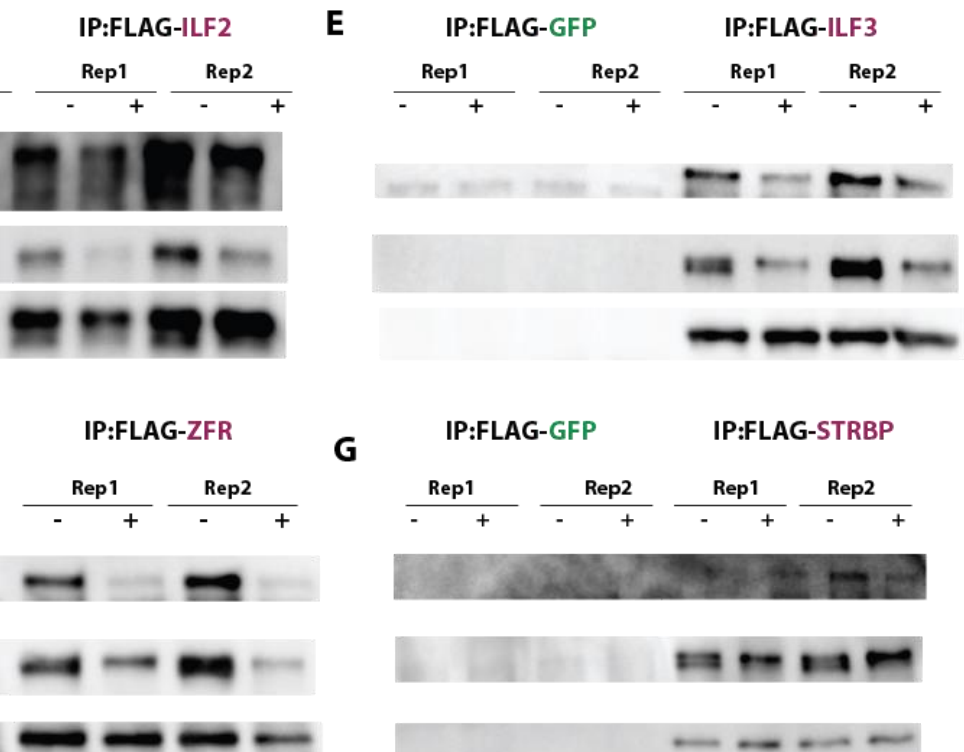

G
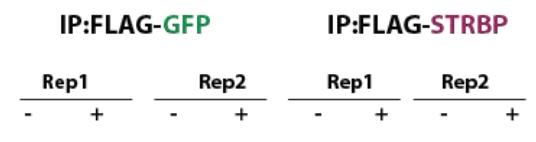

\section{Figure 3. co-IPs demonstrate that DZF-domain-containing BiolD hits interact with ADAR1 and ADAR2 in an RNA-dependent manner}

A. Schematic depicting ADAR1, ADAR2 and DZF-domain-containing proteins ILF2, STRBP, ILF3, and ZFR. Conserved domains are indicated with different colors. B-C. Western blots of FLAG immunoprecipitation of M17 cells overexpressing either FLAG-GFP (negative control), FLAG-ADAR1, or FLAG-ADAR2. IPs were performed with or without the addition of RNase $A$ to the lysates prior to IP, as indicated. D-G. Western blots of FLAG immunoprecipitation of M17 cells overexpressing ADAR2 as well as FLAG-GFP (negative control) or a FLAG tagged version of each DZF-domain-containing protein, (D) ILF2, (E) ILF3, (F) ZFR, (G) STRBP. IPs were performed with or without the addition of RNase A to the lysates prior to IP, as indicated. ILF2, which interacts with itself, ILF3, ZFR, and STRBP in an RNAindependent manner, was used as a positive control. 

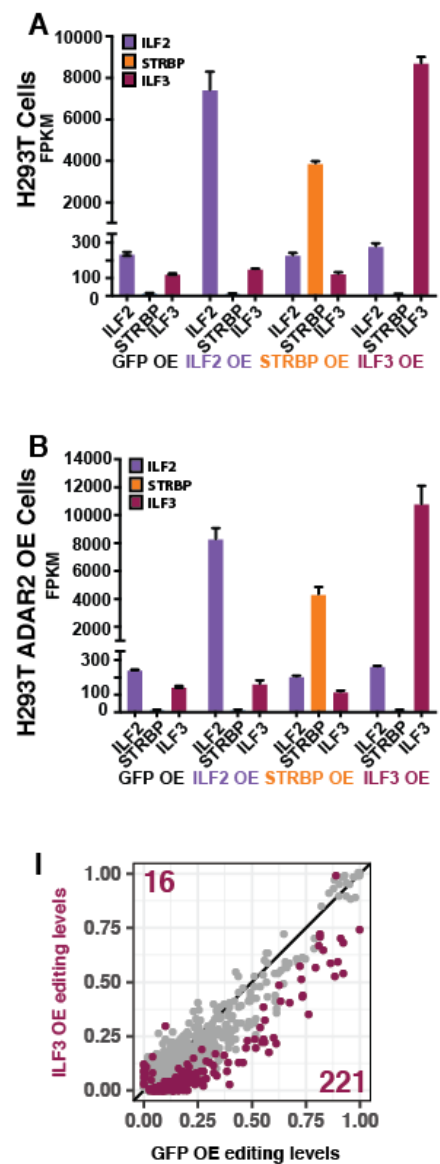

944

945

946

947

948

949

950

951

952

953

954

955

956

957

958

959

960

961

962

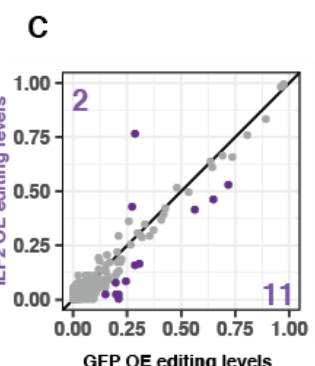

E

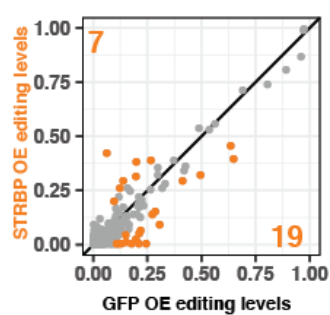

D
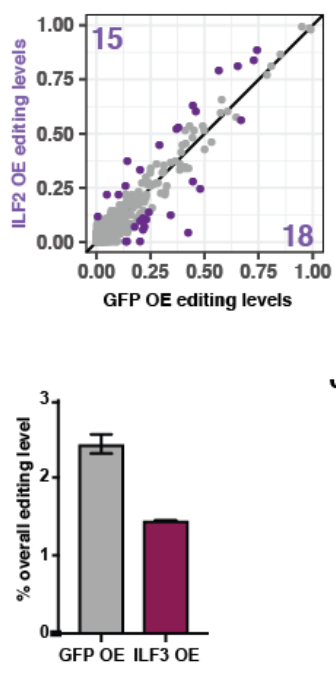

F

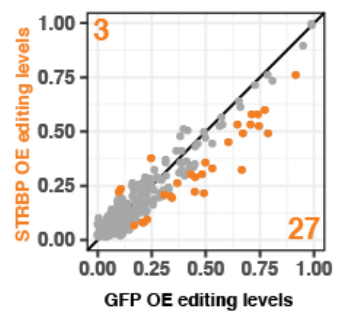

J

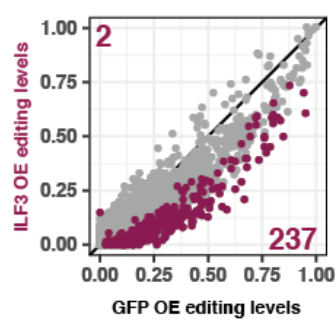

G

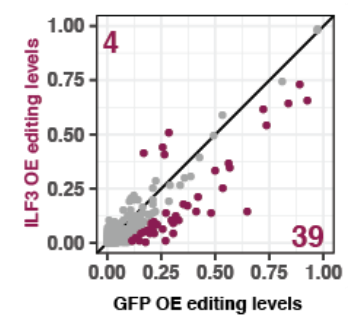

H
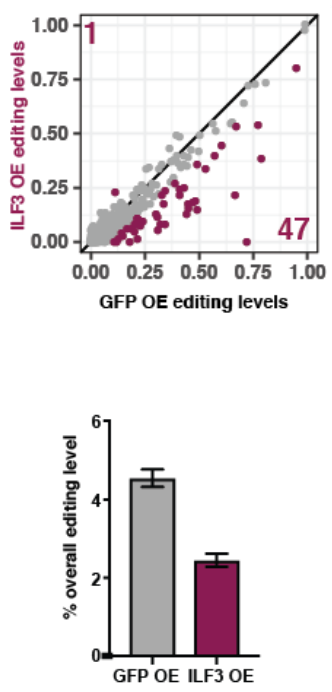

Figure 4. Overexpression of DZF-domain-containing proteins alters RNA editing levels.

A-B. FPKMs of ILF2, STRBP, and ILF3 in (A) HEK293T and (B) HEK293T-ADAR2-overpression cells transiently transfected with GFP, ILF2, STRBP, or ILF3. Error bars represent standard deviation. C-H. Scatterplots comparing RNA editing levels assayed by mmPCR-seq in HEK293T cells (upper) and HEK293T cells stably overexpressing ADAR2 (lower). Plots compare editing levels in cells overexpressing GFP with editing levels in cells overexpressing a DZF-domain-containing protein ILF2 $(C, D)$, STRBP $(E, F)$ ILF3 $(G, H)$. Colored dots indicate sites that are significantly changed $(p<0.05$, Fisher's exact tests). The number of sites with significantly increased (top left) or significantly decreased (bottom right) editing levels are indicated on each graph. I-J. Scatterplots comparing RNA editing levels assayed by RNA-seq in HEK293T cells (I) and HEK293T cells stably overexpressing ADAR2 (J). Plot compares editing levels in cells overexpressing GFP with editing levels in cells overexpressing ILF3. Colored dots indicate sites that are significantly changed $(p<0.05$, Fisher's exact tests). The number of sites with significantly increased (top left) or significantly decreased (bottom right) editing levels are indicated on each graph. Bar graphs to the right of each scatterplot depict overall editing levels (percentage of edited reads at all sites) for GFP overexpression (grey) and ILF3 overexpression (red). 
A

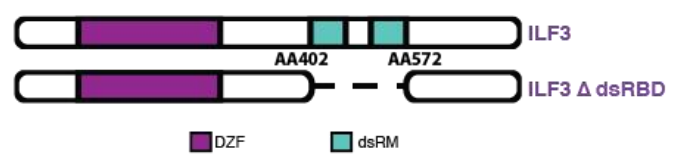

B

H293T Cells

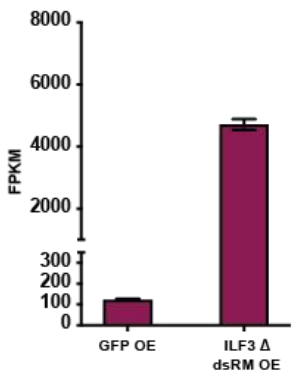

C

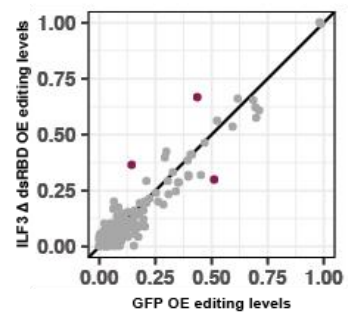

H293T ADAR2 OE Cells
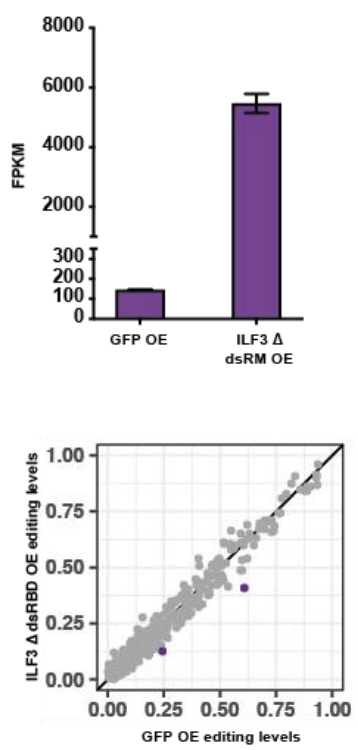

D

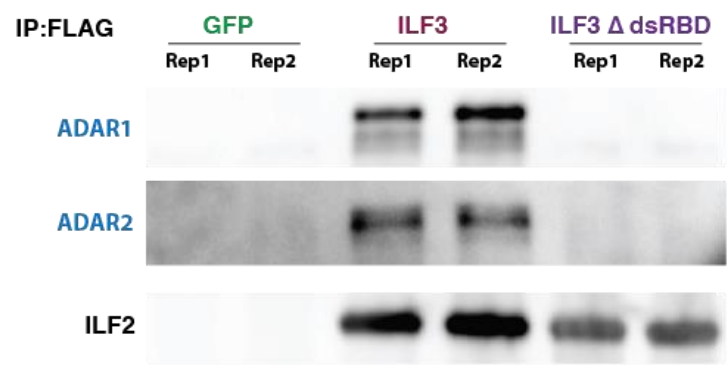

IP:FLAG

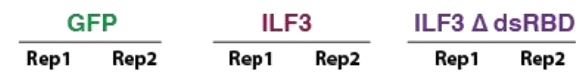

$+\cdots$

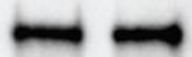

Blot:FLAG

Figure 5. Overexpression of ILF3 dsRNA-binding mutant does not interact with either ADAR or affect editing levels. A. Schematic of ILF3 double-stranded RNA binding domain (dsRBD mutant, dashed lines indicate the deleted region (which spans amino acids 402-572). B. Bar graphs depicting the FPKM of ILF3 in GFP and ILF3 $\triangle$ dsRBD overexpression in HEK293T cells (left) and HEK293T cells stably overexpressing ADAR2 (right). C. Scatterplots comparing RNA editing levels assayed by mmPCR-seq in HEK293T cells (left) and HEK293T cells stably overexpressing ADAR2 (right). Plot compares editing levels in cells overexpressing GFP (negative control, $x$-axis) with editing levels in cells overexpressing ILF3 $\Delta$ dsRBD ( $y$-axis). Colored dots indicate sites that are significantly changed $(p<0.05$, Fisher's exact tests). Overexpression of the ILF3 dsRBD mutant does not affect editing levels. D. Western blots of FLAG immunoprecipitation of HEK293T cells overexpressing ADAR2 and either FLAG-GFP (negative control), FLAG-ILF3, or FLAG- ILF3 $\triangle$ dsRBD. ILF3 $\triangle$ dsRBD mutant does not interact with ADAR1 or ADAR2, but maintains its interaction with ILF2. 
978

979

980

\section{Supplemental Figures}

PAICS - chr4: $57,326,879$

M17 BirA*-GFP

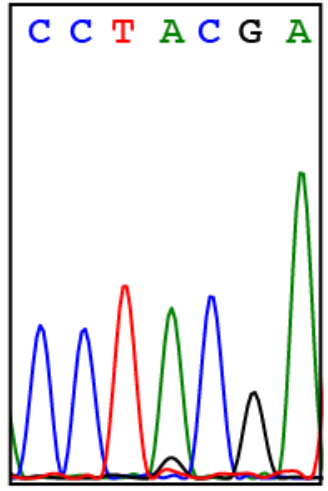

$\uparrow$
M17 BirA*-ADAR1

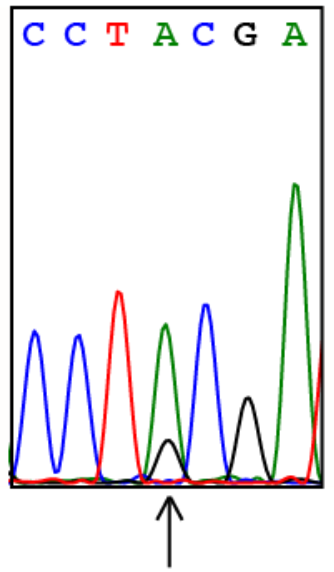

M17 BirA*-ADAR2

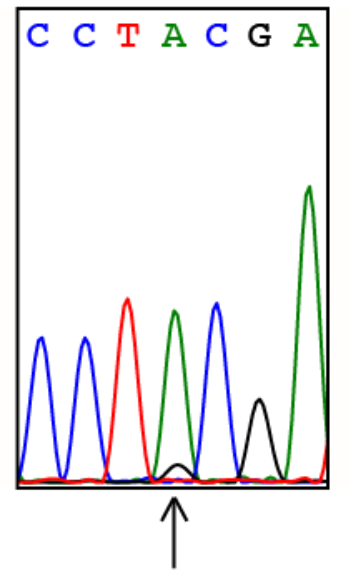

GRIA2 - chr4: 158,257,875

981

982

983

984

985

986

987

988

989

990

991

M17 BirA*-ADAR1 M17 BirA*-ADAR2

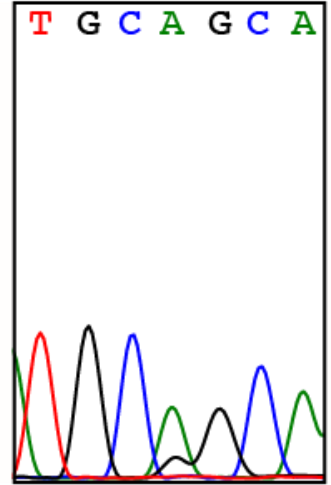

$\uparrow$

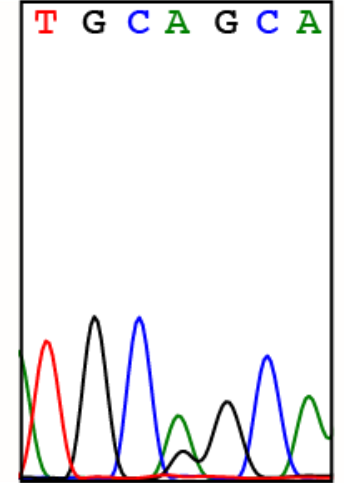

$\uparrow$

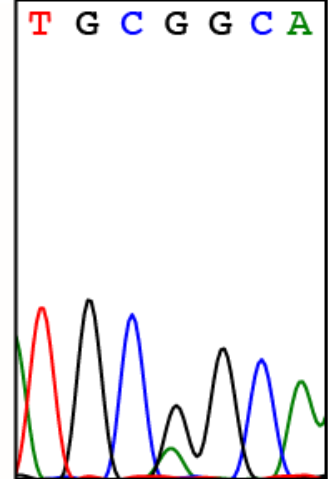

$\uparrow$

Figure S1. BirA*-ADAR1 and BirA*-ADAR2 retain editing activity. (A) Sanger sequencing traces at an ADAR1-regulated editing site in PAICS (chr4: 57,326,879) in M17 cells expressing BirA*-GFP, BirA*ADAR1, and BirA*-ADAR2 assayed in BiolD experiments. The site is most highly edited in cells expressing Bir $A^{*}$-ADAR1. (B) Sanger sequencing traces at an ADAR2-regulated editing site in GRIA2 (chr4: 158,257,875) in M17 cells expressing BirA*-GFP, BirA*-ADAR1, and BirA*-ADAR2 assayed in BiolD experiments. The site is most highly edited in cells expressing Bir $A^{*}-A D A R 2$. Arrows denote each editing site in the sequence. 

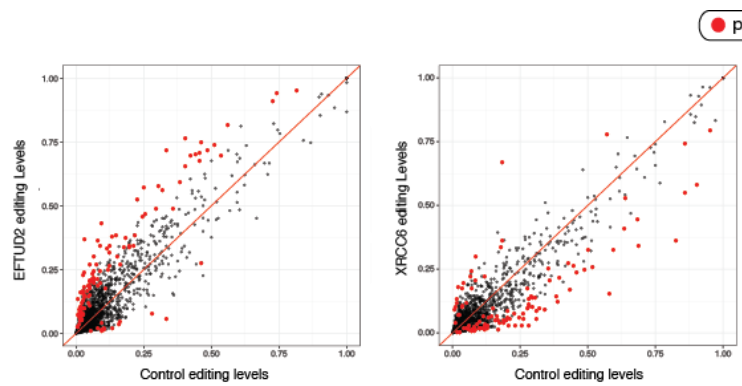

p-value $<0.05$
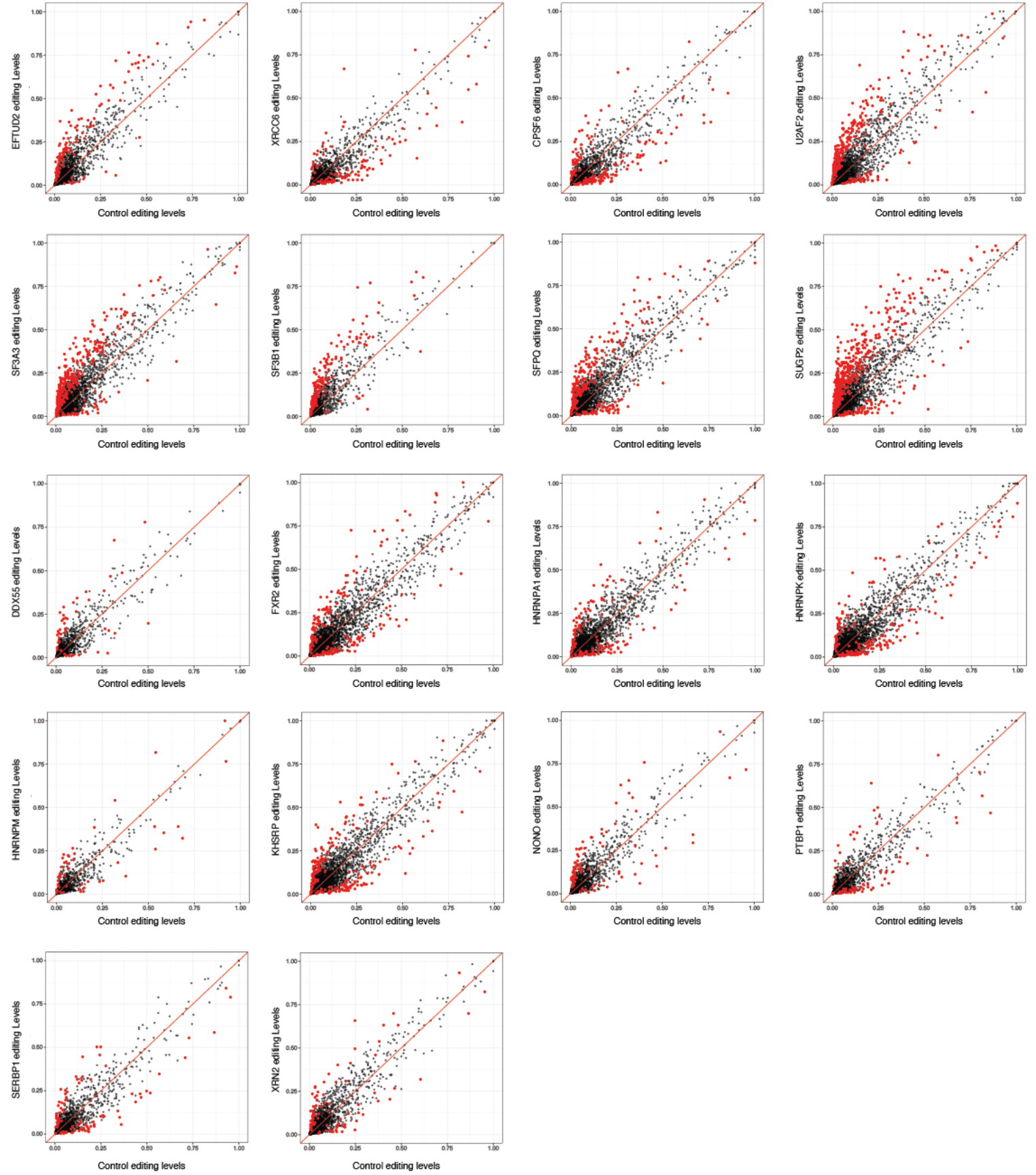

Figure S2. Scatter plots of pairwise comparison of editing levels between knockdown and control RNAseq of RBPs that were also found in the BiolD assay. Red dots, Fisher's exact test $p$-value $<0.05$. 
A

\begin{tabular}{|c|c|c|}
\hline & $\begin{array}{c}\begin{array}{c}\text { \# of } \\
\text { proteins }\end{array} \\
\end{array}$ & \# hits \\
\hline \multirow{2}{*}{$\left.\frac{\pi}{\frac{\pi}{I}}\right|_{\text {ADAR2 }} ^{\text {ADAR1 }}$} & 857 & 26 \\
\hline & 1234 & 143 \\
\hline \multirow{2}{*}{$\left.\bar{\Sigma}\right|_{\text {ADAR2 }} ^{\mathrm{ADAR} 1}$} & 597 & 49 \\
\hline & 750 & 128 \\
\hline
\end{tabular}

B

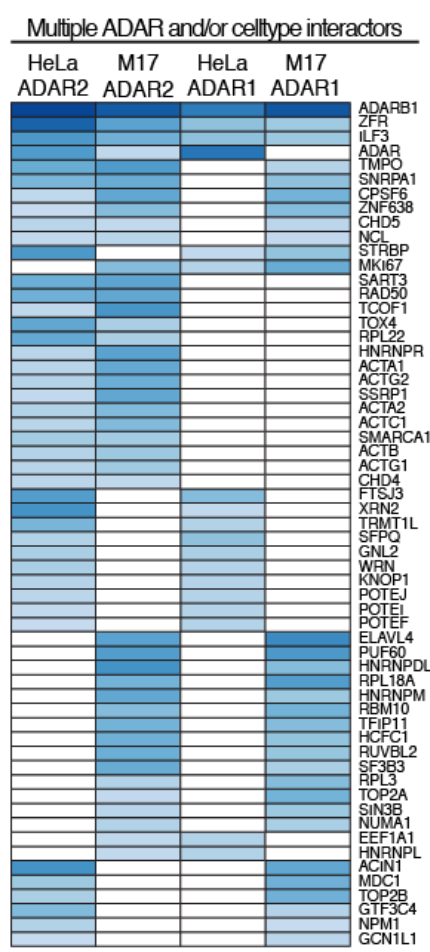

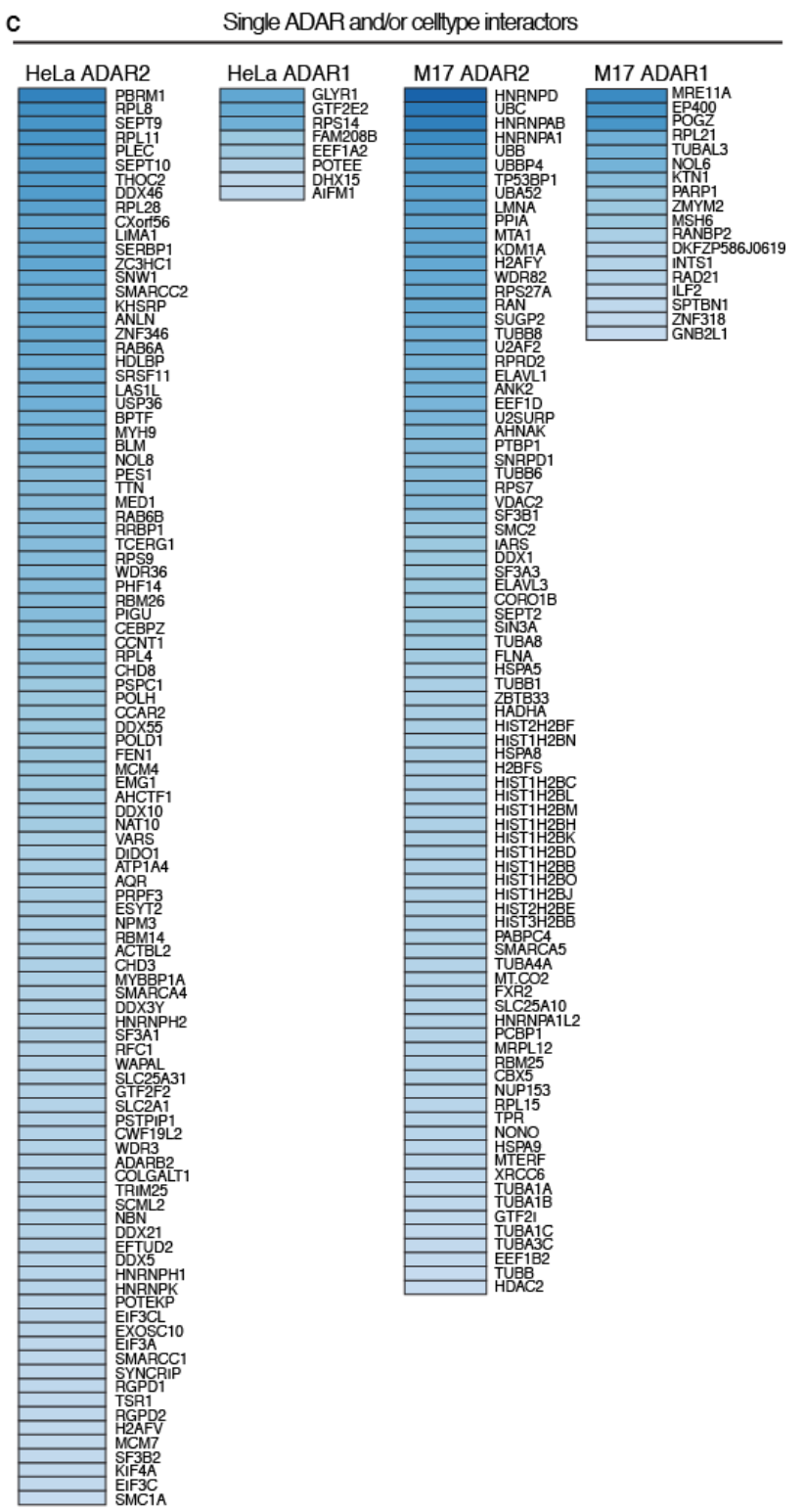

Figure S3. BioID of ADAR1 and ADAR2 in HeLa and M17 cells reveals interactors specific to each cell type and each ADAR (A) The total number of proteins identified by mass spec from each IP and the number of hits remaining after the filtering pipeline illustrated in Figure 1C. (B) A heatmap displaying the hits identified in multiple IPs. All proteins detected in at least two conditions are displayed, arranged by unsupervised hierarchical clustering. The strength of blue indicates the log fold change over the GFP control. White indicates that the protein was not detected in the IP. (C) A heatmap displaying all proteins identified in only one condition. The strength of blue indicates the log fold change over the GFP control. 
A

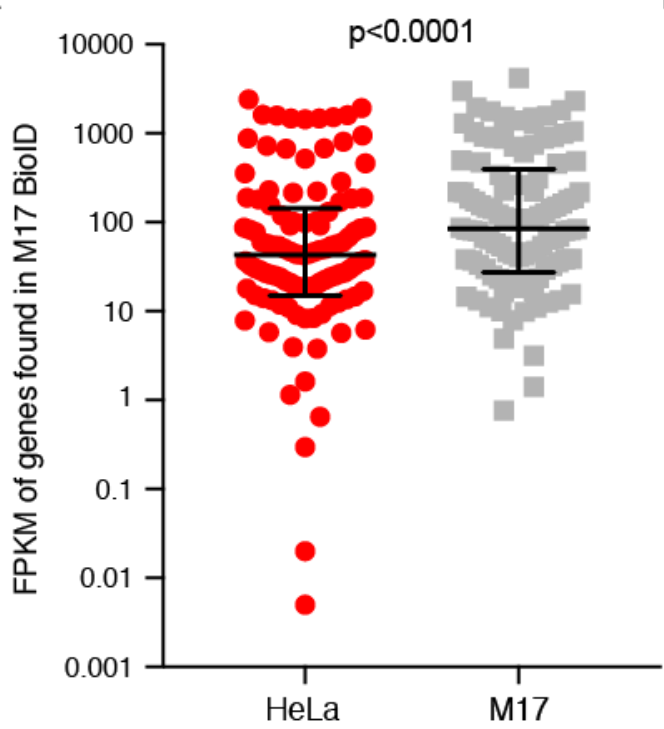

B

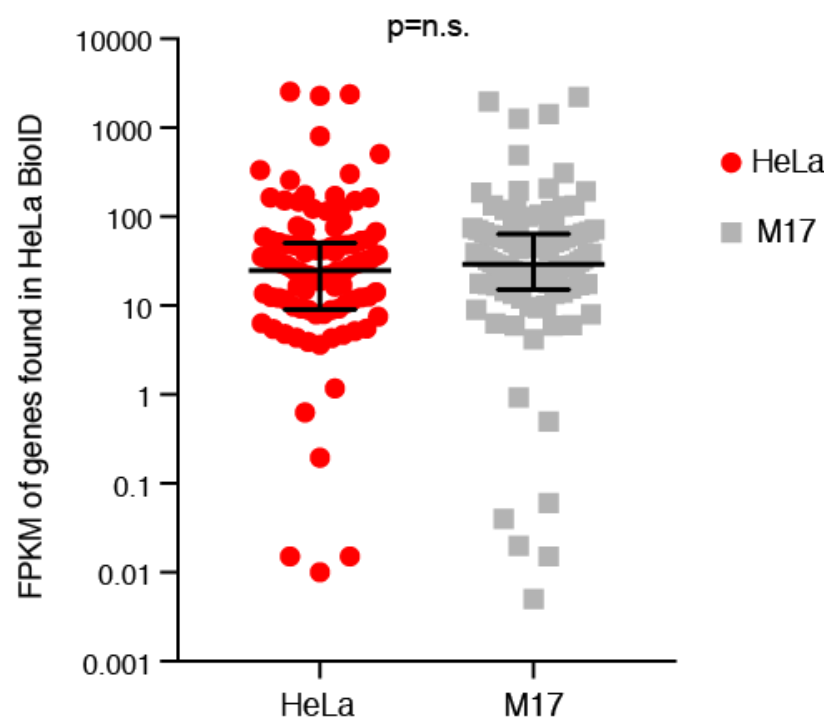

1008

1009

1010

1011

1012

1013

1014

1015

1016

1017
Figure S4. Genes found in the M17 BiolD are more highly expressed in M17 cells. A. Gene expression of all genes that encode proteins identified in the M17 ADAR1 and ADAR2 BioID screens. Each dot represents the FPKM in HeLa (red) or M17 (grey) cells, as assayed by RNA-seq. Overall, the set of genes is more highly expressed in M17 cells versus HeLa. B. Gene expression of all genes that encode proteins that were identified in the HeLa ADAR1 and ADAR2 BioID screens. Each dot represents the FPKM in HeLa (red) or M17 (grey) cells, as assayed by RNA-seq. There is not a significant difference between HeLa and M17. The median (middle black bar) with interquartile range (HeLa, black bars and M17, black bars) are shown for each plot. P-values were determined by Wilcoxon matched pairs signed rank test. 
A

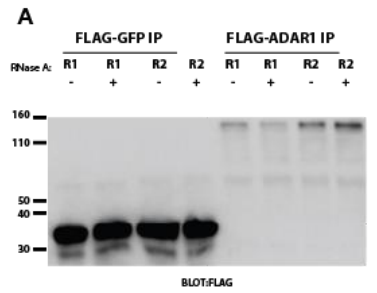

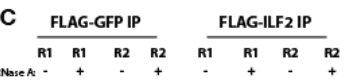

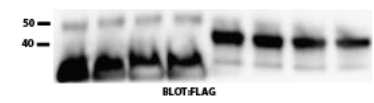

E FLAG-GFPIP FLAG-ZFRIP

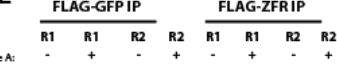

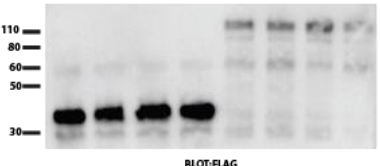

FLAG-GFP Input FLAG-ADAR1 Input \begin{tabular}{lllllllllllllllll}
\hline R1 & R1 & R2 & R2 & R1 & R1 & R2 & R2
\end{tabular}

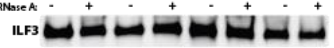
นเ2 ニニニニニニニニ

STRB $=00--\cdots$

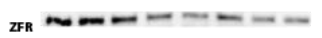

GАРОН - - - - - -

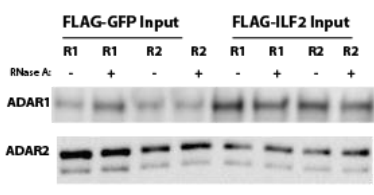

${ }_{\mathrm{GAPH}}-\cdots--\cdots$

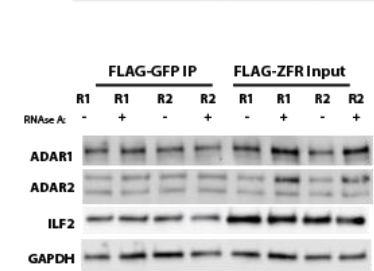

B FLAG-GFP IP FLAG-ADAR2 IP

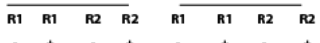

$110-$

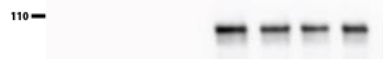

${ }^{50}-$

30-

Btorfinas

D

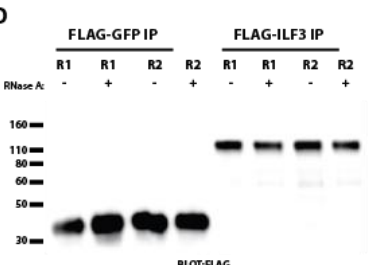

Btorfac

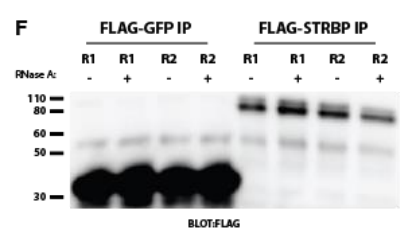

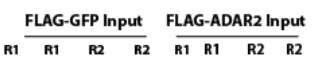

RHosen -

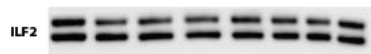

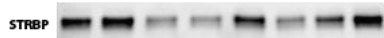

zFR $-\cdots$

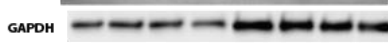

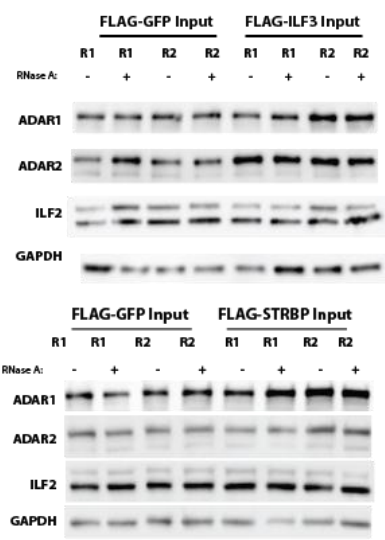

Figure S5. Input of the IPs from Figure 3 show strong expression of each FLAG-tagged construct and blotted protein. A. Inputs of IPs from Figure 3B. B. Inputs of IPs from Figure 3C. C. Inputs of IPs from Figure 3D. D. Inputs of IPs from Figure 3E. E. Inputs of IPs from Figure 3F. F. Inputs of IPs form Figure $3 \mathrm{G}$. 
A

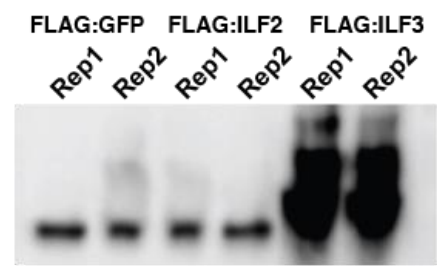

WB:ILF3

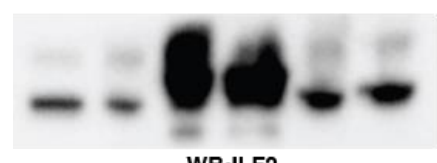

WB:ILF2

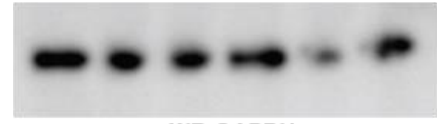

WB:GAPDH
FLAG:GFP FLAG:STRBP
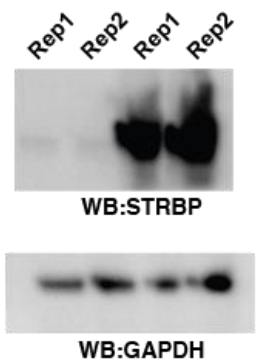

WB:GAPDH

B

HEK293T ADAR2 OE

Figure S6. The overexpression of each DZF-domain-containing protein in the cells lines assayed by RNA-seq and mmPCR-seq in Figure 3 were determined by western blot. A. Protein from HEK293T cells transiently overexpressing FLAG fused to ILF2, ILF3 (left) and STRBP (right) was blotted for ILF3, ILF2, STRBP and GAPDH as a control. B. Protein from HEK293T A2 OE cells transiently overexpressing FLAG fused to ILF2, ILF3 (left) and STRBP (right) was blotted for ILF3, ILF2, STRBP and GAPDH as a control. 


\section{A mmPCR-seq Analysis}
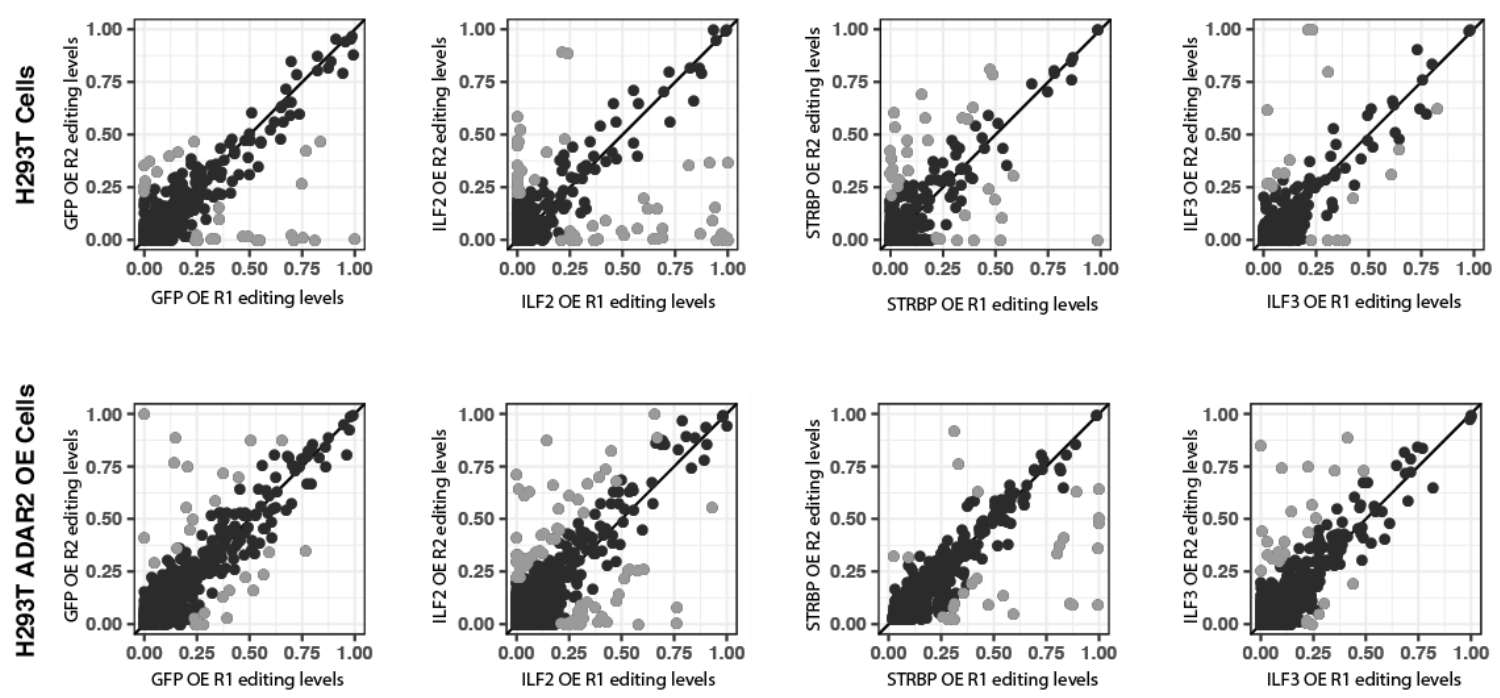

\section{B RNA-seq Analysis}
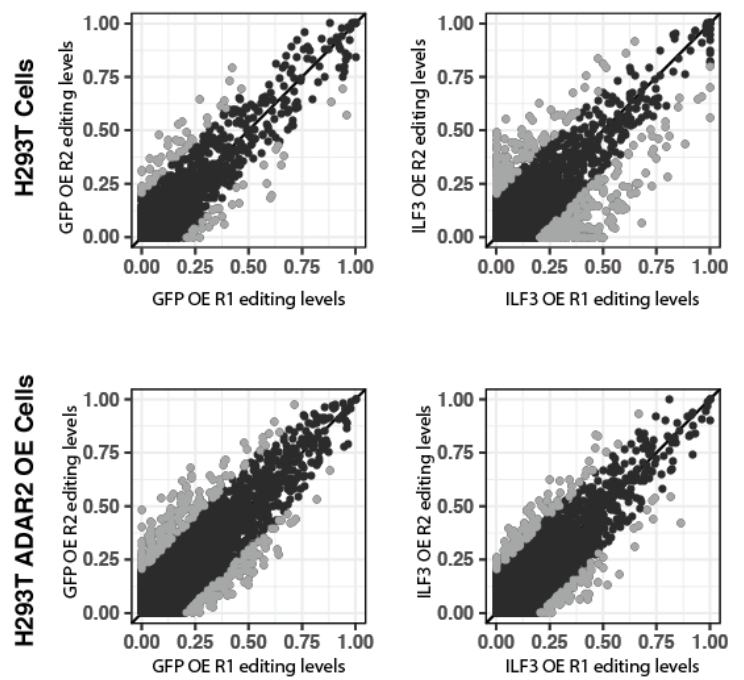

Figure S7. mmPCR-seq to measure editing levels demonstrates low variability in biological replicates. Sites with high variability were removed from further analysis. A. Scatterplots of biological replicates assayed by mmPCR-seq from HEK293T (top row) and HEK293T ADAR2 OE (bottom row) overexpressing GFP, ILF2, STRBP or ILF3 (from Figure 3). Gray dots represent sites with more than $20 \%$ variability between replicates, and were not included in further analysis. B. Scatterplots of biological replicates assayed by RNA-seq from HEK293T (top row) and HEK293T ADAR2 OE (bottom row) overexpressing GFP (left) or ILF3 (right). Gray dots represent sites with more than $20 \%$ variability between replicates, and were not included in further analysis. 
A

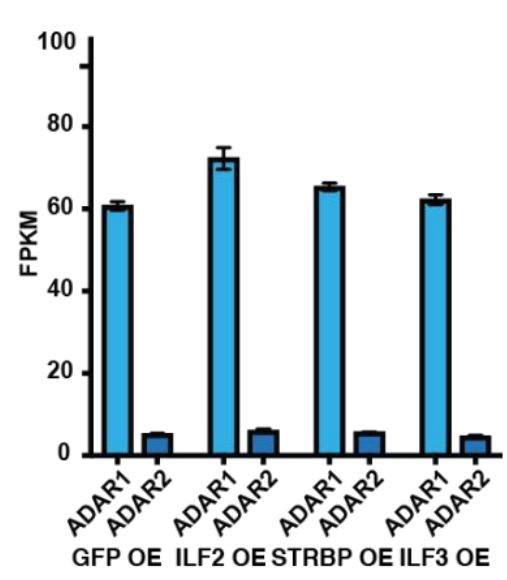

293T ADAR2 OE

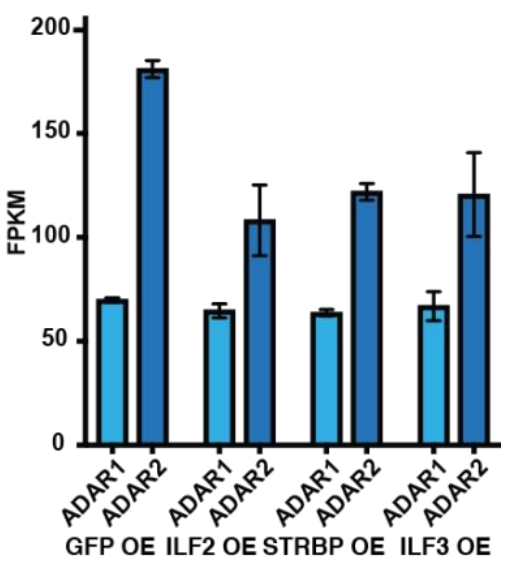

B

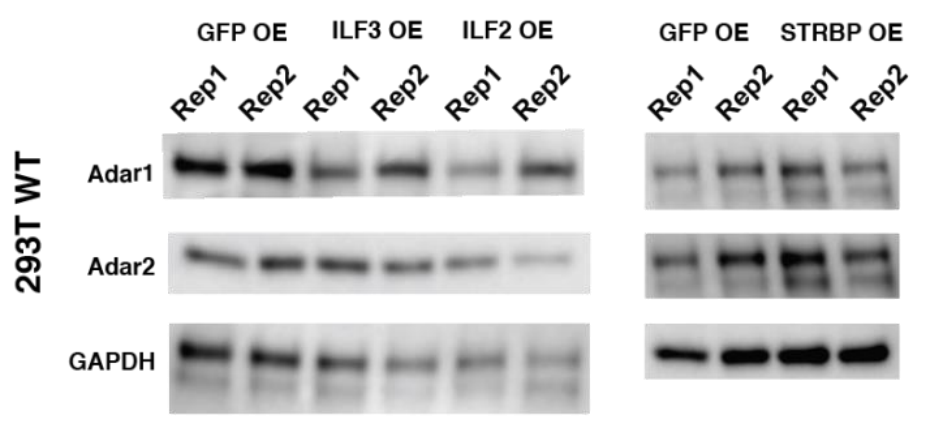

GFP OE ILF3 OE ILF2 OE

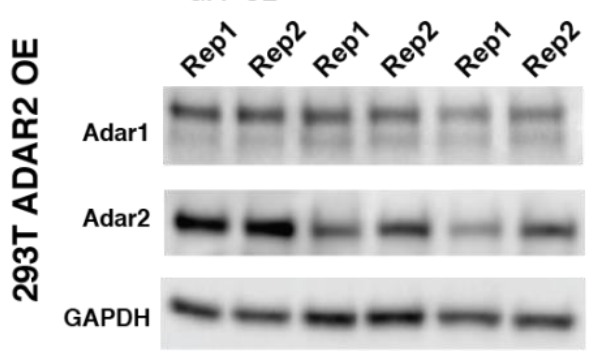

Figure S8. Levels of ADAR1 and ADAR2 are not greatly changed in cells lines overexpressing DZF-domain-containing proteins. A. Levels of ADAR1 (light blue) and ADAR2 (dark blue) in HEK293T WT (left) and HEK293T A2 OE (right) cells overexpressing GFP, ILF2, STRBP or ILF3. $n=2$ biological replicates. The transcript levels of ADAR1 are not significantly changed in HEK293T or HEK293T A2 OE cells. The transcript levels of ADAR2 are reduced in HEK293T A2 OE cells overexpressing DZF-domaincontaining proteins compared to GFP. B. Protein from HEK293T (top) or HEK293T A2 OE (bottom) cells overexpressing GFP, ILF2, ILF3 or STRBP were blotted for ADAR1, ADAR2 and GAPDH as a control. The protein levels of ADAR1 and ADAR2 are not greatly changed in wildtype or HEK293T A2 OE cells. 
A

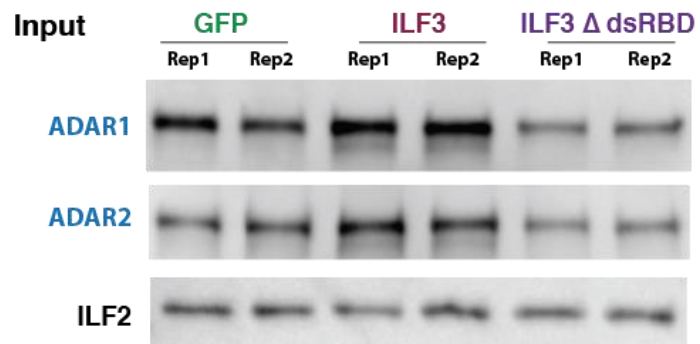

Figure S9. Biological replicates of cells overexpressing ILF3 and ILF3 dsRBD show consistent editing levels. A. Scatterplots of biological replicates assayed by mmPCR-seq from HEK293T (top row) and HEK293T A2 OE (bottom row) overexpressing GFP or ILF3 dsRBD (from Figure 5C). B. Inputs of IPs from Figure 5D.

\section{B}
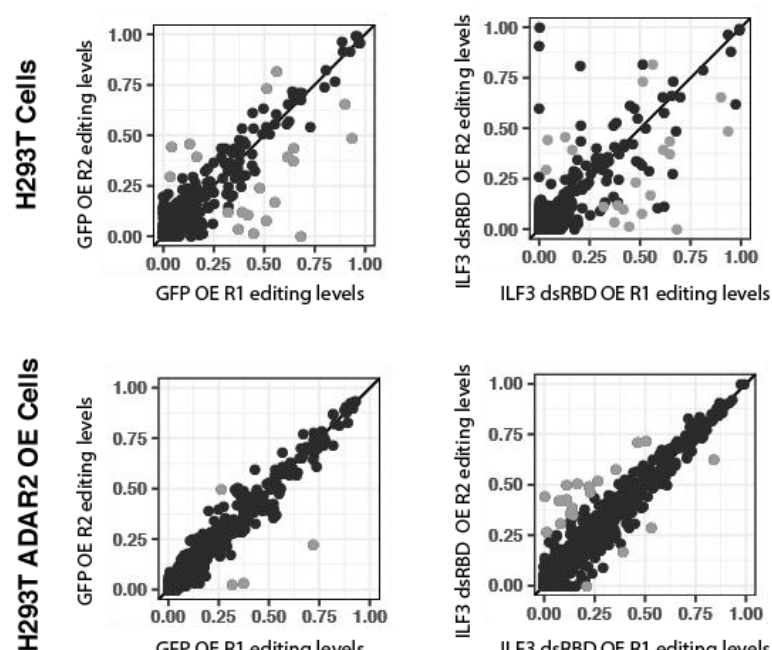

1066 
1067

1068

1069

1070

1071

1072

1073

1074

1075

1076

1077

\section{LIST OF SUPPLEMENTARY TABLES}

Supplementary Table 1. mmPCR-seq of HEK293T and HEK293T Adar2 overexpression with overexpression of ILF2, ILF3, ILF3dsRBD, and STRBP editing levels and p-values for comparison with GFP overexpression controls.

Supplementary Table 2. RNA-seq of HEK293T and HEK293T Adar2 overexpression with ILF3 overexpression editing levels and $p$-values for comparison with GFP overexpression controls. 